\title{
DIRICHLET FORMS AND CONVERGENCE OF BESOV NORMS ON SELF-SIMILAR SETS
}

\author{
Qingsong Gu and Ka-Sing Lau
}

The Chinese University of Hong Kong, Department of Mathematics, Hong Kong and Memorial University of Newfoundland, Department of Mathematics and Statistics NL A1C 5S7, Canada; qsgu@math.cuhk.edu.hk

The Chinese University of Hong Kong, Department of Mathematics, Hong Kong and University of Pittsburgh, Department of Mathematics Pittsburgh, Pa. 15217, U.S.A.; kslau@math.cuhk.edu.hk

\begin{abstract}
Let $B_{2, \infty}^{\sigma}, B_{2,2}^{\sigma}$ denote the Besov spaces defined on a compact set $K \subset \mathbf{R}^{d}$ that is equipped with an $\alpha$-regular measure $\mu$ ( $K$ is called an $\alpha$-set). The critical exponent $\sigma^{*}$ is the supremum of the $\sigma$ such that $B_{2,2}^{\sigma} \cap C(K)$ is dense in $C(K)$. It is known that $B_{2,2}^{\sigma}$ is the domain of a non-local regular Dirichlet form, and for certain standard self-similar set, $B_{2, \infty}^{\sigma^{*}}$ is the domain of a local regular Dirichlet form. In this paper, we study, on the homogenous p.c.f. self-similar sets (which are $\alpha$-sets), the convergence of the $B_{2,2}^{\sigma}$-norm to the $B_{2, \infty}^{\sigma^{*}}$-norm as $\sigma \nearrow \sigma^{*}$ and the associated Dirichlet forms. The theorem extends a celebrate result of Bourgain, Brezis and Mironescu [4] on Euclidean domains, and the more recent results on some self-similar sets [10, 22, 29].
\end{abstract}

\section{Introduction}

Let $K$ be a closed subset in $\mathbf{R}^{d}$ with the Euclidean metric, and let $\mu$ be an $\alpha$-regular measure on $K$, that is, there exists $\alpha>0$ such that for any ball $B(x, r)$ with $0<r<\operatorname{diam}(K)$,

$$
\mu(B(x, r)) \asymp r^{\alpha} .
$$

(Here $f \asymp g$ means there exists constant $C>0$ such that $C^{-1} g \leq f \leq C g$.) We call it an $\alpha$-set $\left(d\right.$-set in [15]). Fix $\sigma>0$, for $u \in L^{2}(K, \mu)$, let

$$
[u]_{B_{2, \infty}^{\sigma}}^{2}:=\sup _{0<r<1} r^{-(2 \sigma+\alpha)} \int_{K} \int_{B(x, r)}|u(x)-u(y)|^{2} d \mu(y) d \mu(x),
$$

and define $B_{2, \infty}^{\sigma}:=\left\{u \in L^{2}(K, \mu):\|u\|_{B_{2, \infty}^{\sigma}}<\infty\right\}$ with norm $\|u\|_{B_{2, \infty}^{\sigma}}:=\|u\|_{2}+$ $[u]_{B_{2, \infty}^{\sigma}}$. Similarly we define the space $B_{2,2}^{\sigma}$ by

$$
[u]_{B_{2,2}^{\sigma}}^{2}:=\int_{0}^{1} \frac{d r}{r}\left(r^{-(2 \sigma+\alpha)} \int_{K} \int_{B(x, r)}|u(x)-u(y)|^{2} d \mu(y) d \mu(x)\right) .
$$

These spaces are Banach spaces, and belong to the family of Besov spaces $B_{p, q}^{\sigma}$, which have been studied in great detail in literature (e.g., $[9,14,15,28]$; note that in [14], the space $B_{p, q}^{\sigma}$ is denoted by $\left.\operatorname{Lip}(\sigma, p, q)\right)$. For an $\alpha$-set $K$, the family of

https://doi.org/10.5186/aasfm.2020.4536

2010 Mathematics Subject Classification: Primary 28A80; Secondary 46E30, 46 E35.

Key words: Besov space, Dirichlet form, $\Gamma$-convergence, Harnack inequality, heat kernel, p.c.f. fractal, resistance network and trace.

The research is supported in part by the HKRGC grant. 
spaces $\left\{B_{2, \infty}^{\sigma}\right\}_{\sigma>0}$ is a decreasing sequence of Banach spaces, and can only consist of constant functions when $\sigma$ is large. We call

$$
\sigma^{*}=\sup \left\{\sigma>0: B_{2, \infty}^{\sigma} \cap C(K) \text { is dense in } C(K)\right\}
$$

the critical exponent of $K$. It is known that for $0<\sigma<\sigma^{*}, B_{2,2}^{\sigma}$ is the domain of a stable-like non-local regular Dirichlet form; and in some known cases, $B_{2, \infty}^{\sigma^{*}}$ is the domain of a local regular Dirichlet form (if it exists), which induces a Laplacian on $K$.

For a domain $D$ in $\mathbf{R}^{d}$ with smooth boundary, $B_{2, \infty}^{1}$ is the Sobolev space $W^{1,2}(D)$ with $[u]_{B_{2, \infty}^{1}}^{2} \asymp \int_{D}|\nabla u(x)|^{2} d x$, and the corresponding Dirichlet form is associated with the classical Laplacian $\Delta$; for $0<\sigma<1, B_{2,2}^{\sigma}$ is the fractional Sobolev space $W^{\sigma, 2}(D)$, and $[u]_{B_{2,2}^{\sigma}}^{2} \asymp \iint_{D \times D}|u(x)-u(y)|^{2}|x-y|^{-(d+2 \sigma)} d x d y$, which is associated with the fractional Laplacian $(-\Delta)^{\sigma}$. The critical exponent is $\sigma^{*}=1$. The following is a fundamental theorem on the relationship of the two norms.

Theorem 1.1. (Bourgain, Brezis and Mironescu [4]) Let $D$ be a bounded domain in $\mathbf{R}^{d}$ with smooth boundary, then for $u \in L^{2}(D)$,

$$
\lim _{\sigma \nearrow 1}(1-\sigma) \iint_{D \times D} \frac{|u(x)-u(y)|^{2}}{|x-y|^{d+2 \sigma}} d x d y=C_{d} \int_{D}|\nabla u(x)|^{2} d x
$$

For self-similar sets, it is well-known that the Sierpinski gasket has $\sigma^{*}=\frac{\log 5}{2 \log 2}$ [14]. The exponent is also known for certain simple nested fractals, and an estimation for the Sierpinski carpet [2]. In [11], the authors gave a preliminary study of the critical exponent of some non-standard post critically finite (p.c.f.) self-similar sets. In general there is not much information about the critical exponent, nor the associated Dirichlet form at $\sigma^{*}$.

In [22], Pietruska-Pałuba extended Theorem 1.1 on some nested fractals $K$ through a probabilistic approach, under the assumption that the diffusion process has a sub-Gaussian heat kernel, then approximate by a sequence of subordinate stable-like processes. In an attempt to find a unified analytic approach to study the Laplacian on p.c.f. self-similar sets and non-p.c.f. sets, recently Grigor'yan and Yang [10, 29] proved a discrete analog of (1.4) on the Sierpinski gasket and the Sierpinski carpet, using the $\Gamma$-convergence on the $B_{2,2}^{\sigma}$-norm to $B_{2, \infty}^{\sigma^{*}}$-norm. It is the main purpose of this paper to continue this investigation to extend to certain p.c.f. self-similar sets. The consideration of non-p.c.f. self-similar sets will be in an upcoming paper [12].

Throughout the paper, unless otherwise specified, we always assume that $K$ is a homogeneous connected p.c.f. self-similar set [16] (see (2.1), (2.2)), and for brevity, we just say $K$ is a p.c.f. self-similar set. Note the $K$ is equipped with a normalized $\alpha$-Hausdorff measure $\mu$, and is hence an $\alpha$-set.

Let $V_{0}$ be the boundary of $K, F_{\omega}=F_{i_{1}} \circ \cdots \circ F_{i_{j}}$ where $\omega=i_{1} \cdots i_{j}$, and let

$$
E_{j}[u]=\sum_{x, y \in F_{\omega}\left(V_{0}\right) ;|\omega|=j}|u(x)-u(y)|^{2}
$$

be the energy of $u$ on the $j$-level approximation. First, we need a discretization of the Besov semi-norm $[u]_{B_{2, \infty}^{\sigma}}$.

Theorem 1.2. Let $K$ be a p.c.f. self-similar set. Then for $2 \sigma>\alpha$,

$$
[u]_{B_{2, \infty}^{\sigma}}^{2} \asymp \sup _{j \geq 0}\left\{\rho^{-(2 \sigma-\alpha) j} E_{j}[u]\right\} .
$$


Moreover, for any $\eta>\alpha / 2$, there is a uniform constant $C>0$ for all $\sigma>\eta$ so that the above "ऽ" holds.

The first part of the theorem was first proved by Jonsson in [14] for the Sierpinski gasket. In [3], Bodin extended this discretization theorem to $B_{p, q}^{\sigma}, 0 \leq p, q<\infty$ for the $\alpha$-sets that admit a regular triangular system; for $p$ or $q$ equals $\infty$, he claimed that the proof is similar, and the results apply to the p.c.f. sets directly. However, it seems that there are some technical steps that need to be justified (see Remarks 1, 2 in Section 3). We prove two basic results on the geometry of self-similar sets to overcome the gaps (Section 2 and Appendix section). We will also give a detailed proof of the theorem similar to $[14,3]$ for completeness, and for observing the estimated constants for the $\sigma$ to be uniform as in the second part of the theorem, which will be used in the proof of Theorem 1.4.

The discretization of the space $B_{2,2}^{\sigma}$ can be obtained similarly.

Proposition 1.3. With the same $K$ as in Theorem 1.2, and for $2 \sigma>\alpha$,

$$
[u]_{B_{2,2}^{\sigma}}^{2} \asymp \sum_{j=0}^{\infty} \rho^{-(2 \sigma-\alpha) j} E_{j}[u] .
$$

We remark that in [25], Strichartz constructed several types of functional spaces (including the Besov type) on p.c.f. self-similar sets admitting a regular harmonic structure, in particular, on the Sierpinski gasket. His construction of the Besov spaces uses the discrete approximation of the resistance network of the self-similar energy identity [16]. It is quite different from the approach here on discretizing the continuous Besov spaces, where the regular harmonic structure is not assumed.

Our main objective is to prove the following convergence theorem on Dirichlet forms as in Theorem 1.1 and in [10, 29, 22]. For $2 \sigma-\alpha>0$ and $\sigma<\sigma^{*}$, the above sum defines a non-local regular Dirichlet form on $K$ by polarization, denoted by $\mathcal{E}_{2,2}^{\sigma}$, which has $B_{2,2}^{\sigma}$ as domain. We also define a property (E) (Definition 5.1), which is motivated by a "weak monotone" condition in [10, 29].

Theorem 1.4. Suppose $K$ has property $(E)$. Then $K$ admits a self-similar local regular Dirichlet form $\left(\mathcal{E}^{\sigma^{*}}, \mathcal{F}\right)$ on $K$ with domain $\mathcal{F}=B_{2, \infty}^{\sigma^{*}}$, and there exists $C>0$ such that for $u \in \mathcal{F}$,

$$
C^{-1} \mathcal{E}^{\sigma^{*}}[u] \leq \underline{\lim }_{\sigma \uparrow \sigma^{*}}\left(\sigma^{*}-\sigma\right) \mathcal{E}_{2,2}^{\sigma}[u] \leq \varlimsup_{\sigma \uparrow \sigma^{*}}\left(\sigma^{*}-\sigma\right) \mathcal{E}_{2,2}^{\sigma}[u] \leq C \mathcal{E}^{\sigma^{*}}[u] .
$$

The proof of the theorem makes use of the $\Gamma$-convergence of closed forms in the wide sense $([5,20]$, see also Definition 2.2$)$. The first attempt to use the $\Gamma$-limits to study a diffusive Dirichlet form can be found in [17]. Their setup is on a general metric measure space with volume doubling and some other assumptions; the $\Gamma$ convergence is based on a two-sided heat kernel of the Dirichlet form, discretizing the metric space and the heat kernel to obtain an approximating sequence.

A sufficient condition for property (E) is given by the trace of resistance $R_{n}(p, q)$, $p, q \in V_{0}$ of the electrical network from the discrete energy $E_{n}[u]$.

Theorem 1.5. Suppose there exits $\lambda>1$ and $C>0$ such that for any two distinct points $p, q \in V_{0}$, and for any $n \geq 1, R_{n}(p, q) \asymp \lambda^{n}$. Then $K$ has property (E).

The proof of the theorem makes use of a uniform Harnack inequality of harmonic extensions due to [10] (see Lemma 6.2, Proposition 6.3). In the standard fractals, it is obvious that the sufficient condition is satisfied as $R_{n}(p, q)=\rho^{-n}$ where $\rho$ is the 
renormalizing factor $[16,26]$. We also provide some examples that strict inequality in the condition holds.

For the organization of the paper, we state some definitions and basic results in Section 2, and leave the proof of the new results in the Appendix section, as the techniques are quite different from the main theme of the paper. We prove the discretization of $B_{2, \infty}^{\sigma}$ and $B_{2,2}^{\sigma}$ in Sections 3 and 4 . We introduce property (E) and prove Theorem 1.4 in Section 5, and Theorem 1.5 in Section 6. Section 7 is for some examples and remarks.

\section{Preliminaries}

We first recall the definition of a Dirichlet form. Let $(M, d)$ be a locally compact, separable metric space, and let $\nu$ be a Radon measure on $M$ with $\operatorname{supp}(\nu)=M$. Let $C_{0}(M)$ denote the space of continuous functions with compact support. A closed form in $L^{2}(M, \nu)$ is a symmetric bilinear form that is non-negative definite, closed, densely defined on $L^{2}(M, \nu)$. We say a closed form in $L^{2}(M, \nu)$ is in the wide sense if all conditions for a closed form are satisfied except that it may not be densely defined in $L^{2}(M, \mu)$; and we can simply extend the form on $L^{2}(M, \mu)$ by setting value $+\infty$ outside its domain.

Definition 2.1. On $(M, d, \nu)$, a Dirichlet form $\mathcal{E}$ with domain $\mathcal{F}$ is a closed form and satisfies the Markovian property: $u \in \mathcal{F} \Rightarrow \tilde{u}:=(u \vee 0) \wedge 1 \in \mathcal{F}$ and $\mathcal{E}[\tilde{u}] \leq \mathcal{E}[u]$. (Here $\mathcal{E}[u]:=\mathcal{E}(u, u)$ denote the energy of $u$.) It is called regular if $\mathcal{F} \cap C_{0}(M)$ is dense in $C_{0}(M)$ with the supremum norm, and dense in $\mathcal{F}$ with the $\mathcal{E}_{1}^{1 / 2}$-norm. It is called local if $\mathcal{E}(u, v)=0$ for $u, v \in \mathcal{F}$ having disjoint compact supports.

Note that for $\sigma<\sigma^{*}$, the quadratic form in (1.3) always defines a non-local regular Dirichlet form with $B_{2,2}^{\sigma}$ as domain. On the other hand in the known cases, if (1.2) defines a local regular Dirichlet form, then the domain is $B_{2, \infty}^{\sigma^{*}}$; it is a nontrivial matter to construct or to prove the existence of such form. We will consider this problem through the $\Gamma$-convergence [5].

Definition 2.2. Let $\mathcal{E}^{n}$ and $\mathcal{E}$ be closed forms on $L^{2}(M, \nu)$ in the wide sense. We say that $\mathcal{E}^{n} \Gamma$-converges to $\mathcal{E}$ if

(i) for any $\left\{u_{n}\right\}_{n=1}^{\infty} \subset L^{2}(M, \nu)$ converging strongly to $u, \underline{\lim }_{n \rightarrow \infty} \mathcal{E}^{n}\left[u_{n}\right] \geq \mathcal{E}[u]$;

(ii) for any $u \in L^{2}(M, \nu)$, there exists a sequence $\left\{u_{n}\right\} \subset L^{2}(M, \nu)$ converges strongly to $u$ such that $\varlimsup_{n \rightarrow \infty} \mathcal{E}^{n}\left[u_{n}\right] \leq \mathcal{E}[u]$.

Theorem 2.3. ([5, Theorem 8.5], [20, Theorem 2.8.1]) Let $\left\{\mathcal{E}^{n}\right\}_{n}$ be a sequence of closed forms in the wide sense on $L^{2}(M, \nu)$, then there exists a subsequence $\left\{\mathcal{E}^{n_{k}}\right\}_{k}$ that $\Gamma$-converges to a closed form $\mathcal{E}$ in the wide sense on $L^{2}(M, \nu)$.

Let $\left\{F_{i}\right\}_{i=1}^{N}$ be an IFS of similitudes on $\mathbf{R}^{d}$ with contraction ratios $\left\{\rho_{i}\right\}_{i=1}^{N}$. Let $K=\bigcup_{i=1}^{N} F_{i}(K)$ be the self-similar set; a self-similar measure $\mu$ is a probability measure defined by $\mu=\sum_{i=1}^{N} p_{i} \mu \circ F_{i}^{-1}$ where $\left\{p_{i}\right\}_{i=1}^{N}$ is a set of probability weights. Note that the support of $\mu$ is $K$. An IFS is said to satisfy the open set condition (OSC) if there is a bounded nonempty open set $U$ such that $F_{i}(U) \subset U$, and $F_{i}(U) \cap F_{j}(U)=$ $\emptyset$ for $i \neq j$. In this case $\operatorname{dim}_{H}(K):=\alpha$, the Hausdorff dimension of $K$, is given by $\sum_{i=1}^{N} \rho_{i}^{\alpha}=1$; also if we take $p_{i}=\rho_{i}^{\alpha}$ (the natural weight), then $\mu$ is the normalized Hausdorff measure $\mathcal{H}^{\alpha}$ on $K$.

We define the symbolic space as usual. Let $\Sigma=\{1, \cdots, N\}$ be the alphabets, $\Sigma^{n}$ the set of words $\omega=i_{1} \cdots i_{n}$ of length $n=:|\omega|$, and $\Sigma^{\infty}$ the set of infinite words 
$\omega=i_{1} i_{2} \cdots$. We write $F_{\omega}=F_{i_{1}} \circ \cdots \circ F_{i_{n}}$, and $K_{\omega}=F_{\omega}(K)$. Let $\pi: \Sigma^{\infty} \rightarrow K$ be defined by $\{x\}=\{\pi(\omega)\}=\bigcap_{n>1} K_{i_{1} \cdots i_{n}}$, a symbolic representation of $x \in K$ by $\omega$.

The following proposition is kindly provided by Professor D.-J. Feng. We will give his proof in the Appendix section.

Proposition 2.4. (Feng [8]) For any IFS $\left\{F_{i}\right\}_{i=1}^{N}$ of similitudes on $\mathbf{R}^{d}$, let $\mu$ be a self-similar measure on $K$. Suppose $K$ is not contained in a hyperplane, then for any ball $B(x, r) \subset \mathbf{R}^{d}, \mu(\partial B(x, r))=0$.

In this paper we only consider IFS $\left\{F_{i}\right\}_{i=1}^{N}$ of similitudes that are homogenous, i.e.,

$$
F_{i}(x)=\rho\left(x-b_{i}\right)+b_{i}, \quad 1 \leq i \leq N .
$$

If the IFS satisfies the OSC, then $\operatorname{dim}_{H}(K)=\alpha=-\log N / \log \rho$. Following Kigami [16], we define the critical set $\mathcal{C}$ and the post-critical set $\mathcal{P}$ for $K$ by

$$
\mathcal{C}=\pi^{-1}\left(\bigcup_{1 \leq i<j \leq N}\left(K_{i} \bigcap K_{j}\right)\right), \quad \mathcal{P}=\bigcup_{m \geq 1} \tau^{m}(\mathcal{C})
$$

where $K_{i}=F_{i}(K), \tau: \Sigma^{\infty} \rightarrow \Sigma^{\infty}$ is the left shift by one index. If $\mathcal{P}$ is a finite set, we call $\left\{F_{i}\right\}_{i=1}^{N}$ a post-critically finite (p.c.f.) IFS, and $K$ is a p.c.f. self-similar set. The boundary of $K$ is defined to be $V_{0}=\pi(\mathcal{P})$. We also define

$$
V_{n}=\bigcup_{i \in\{1, \ldots, N\}} F_{i}\left(V_{n-1}\right), \quad V_{*}=\bigcup_{n \geq 1} V_{n} .
$$

It is clear that $K$ is the closure of $V_{*}$. We call $V_{\omega}:=F_{\omega}\left(V_{0}\right)$ a cell of $V_{n}$ for any $\omega \in \Sigma^{n}$.

For brevity, by a p.c.f. self-similar set $K$, we will mean the $K$ is generated by a homogeneous p.c.f.; we also assume that $K$ is connected and $\#\left(V_{0}\right) \geq 2$ to avoid triviality. It is known that a p.c.f. IFS in (2.1) satisfies the open set condition [6], hence $K$ admits an $\alpha$-Hausdorff measure and is an $\alpha$-set.

Remark. More generally, the OSC holds if the associated similar matrices $A_{i}$ of the p.c.f. $\left\{F_{i}\right\}_{i=1}^{N}$ are commensurable (instead of the $\rho$ in (2.1)), i.e., there exists $A$ such that $A_{i}=A^{n_{i}}$. But it is not true without the commensurable assumption [27].

We will need another type of separation property of the IFS. We say that an IFS (or $K$ ) satisfies condition $(\mathrm{H})$ if

(H) there exists $c_{0}>0$ such that for any integer $m \geq 1$ and any two words $\omega$ and $\omega^{\prime}$ with length $m$ and $K_{\omega} \cap K_{\omega^{\prime}}=\emptyset$ (if and) only if $\operatorname{dist}\left(K_{\omega}, K_{\omega^{\prime}}\right) \geq c_{0} \rho^{m}$. Equivalently, $|x-y|<c_{0} \rho^{n}$ (if and) only if $x$ and $y$ lie in the same or neighboring $n$-cells.

The property on the Sierpinski gasket is obvious and was used in [14]. It was also studied in [19] in another context, and there are non-p.c.f. examples that condition (H) fails even with the open set condition. In [23], Pietruska-Pałuba and Stós introduced another separation condition called property $(\mathrm{P})$ which is similar to property $(\mathrm{H})$. They proved that it holds on certain nested fractals, and was used to study the Hajłasz-Sobolev spaces on nested fractals via the Poincaré inequality.

Proposition 2.5. Suppose the IFS $\left\{F_{i}\right\}_{i=1}^{N}$ in (2.1) has the p.c.f. property, then it satisfies condition $(H)$.

We will give a full proof of the proposition in the Appendix section. It will be used in Section 3 for the discretization of the Besov norm (Lemma 3.3), and in the proof of Theorem 1.5 in Section 6. 


\section{Discretizing $\boldsymbol{B}_{2, \infty}^{\sigma}$}

In this section, we will provide a discrete expression of (1.2) on a p.c.f. self-similar set $K$. The main idea of proof is similar to Jonsson [14] on the Sierpinski gasket, together with Propositions 2.4 and 2.5. We first recall a well-known result (c.f. for example [9]).

Proposition 3.1. Let $K$ be an $\alpha$-set. For $2 \sigma>\alpha$, the identity map $\iota: B_{2, \infty}^{\sigma} \rightarrow$ $C^{(2 \sigma-\alpha) / 2}(K)$ is a continuous embedding with $|u(x)-u(y)| \leq C|x-y|^{(2 \sigma-\alpha) / 2}\|u\|_{B_{2, \infty}^{\sigma}}$ for some $C>0$. (Here $C^{\beta}(K)$ denotes the class of Hölder continuous functions of order $\beta$ on $K$.)

(Note that in [9], the proposition is stated under the assumption that a heat kernel exists, but it was not used in the proof.) It follows that for $2 \sigma>\alpha$, all the functions in $B_{2, \infty}^{\sigma}$ are continuous.

It is easy to see that the semi-norm $[u]_{B_{2, \infty}^{\sigma}}$ has an equivalent expression

$$
[u]_{B_{2, \infty}^{\sigma}}^{2} \asymp \sup _{j \geq 0} r^{-(2 \sigma+\alpha) j} \int_{K} \int_{B\left(x, c r^{j}\right)}|u(x)-u(y)|^{2} d \mu(y) d \mu(x),
$$

where $j$ is an integer, and $c>0,0<r<1$ are any fixed constants. On the p.c.f. set $K$, for simplicity we will take $r=\rho$ and $c$ to be the constant $c_{0}$ in the definition of condition (H). Let $V_{n}=\bigcup\left\{V_{\omega}:|\omega|=n\right\}$ and let $\mu_{n}=\frac{1}{\left|V_{n}\right|} \sum_{p \in V_{n}} \delta_{p}$ where $\delta_{p}$ is the Dirac measure at point $p$, then $\mu_{n}$ weak $^{*}$-converges to $\mu$. Let

$$
I_{n, j}=\int_{K} \int_{B\left(x, c \rho^{j}\right)}|u(x)-u(y)|^{2} d \mu_{n}(y) d \mu_{n}(x) .
$$

Lemma 3.2. On a p.c.f. self-similar set $K$,

$$
\lim _{n \rightarrow \infty} I_{n, j}=\int_{K} \int_{B\left(x, c \rho^{j}\right)}|u(x)-u(y)|^{2} d \mu(y) d \mu(x) .
$$

Remark 1. In [3], the continuity of $|u(x)-u(y)|^{2}$ on $K \times K$ was used in applying the weak ${ }^{*}$-convergence of $\mu_{n}$ to $\mu$. In fact we need to consider the discontinuous $\chi_{B\left(x, c \rho^{j}\right)}|u(x)-u(y)|^{2}$ and apply Proposition 2.4 for such conclusion.

Proof. It is well-known that if a sequence of bounded regular Borel measures $\left\{\nu_{n}\right\}_{n}$ converges to $\nu$ weakly on a compact Hausdorff space $E$, then $\lim _{n \rightarrow \infty} \int_{E} f d \nu_{n}=$ $\int_{E} g d \nu$ for any Borel measurable $f$ such that the set of discontinuity of $g$ is a $\nu$-zero set. By Proposition 3.1, $u$ is continuous; letting $f(x, y)=\chi_{B\left(x, c \rho^{j}\right)}(y) \cdot|u(x)-u(y)|^{2}$, then the set of discontinuity points of $f$ is the set $E=\bigcup_{x \in K}\left(\{x\} \times \partial B\left(x, c \rho^{j}\right)\right)$. By Proposition $2.4, \mu(\partial B(x, r))=0$ for any $r>0$. It follows that $(\mu \times \mu)(E)=0$, and hence by using the weak convergence of $\mu_{n} \times \mu_{n}$ to $\mu \times \mu$, the lemma follows.

Lemma 3.3. Let $K$ be a p.c.f. set. Then for $2 \sigma>\alpha$, there exists $C>0$ such that for any $u \in B_{2, \infty}^{\sigma}$,

$$
I_{n, j} \leq C \rho^{2 \alpha j} \sum_{k=j}^{n} E_{k}[u]
$$

where $E_{k}[u]=\sum_{x, y \in V_{\omega},|\omega|=k}|u(x)-u(y)|^{2}$.

Remark 2. In [3, Theorem 3.23], it was claimed that the Besov space $B_{p, q}^{\sigma}$ on p.c.f. sets can be obtained by the same proof as the regular triangulation setup. However, it seems that the $\mathrm{T} 4$ condition in the regular triangulation has not been 
justified for the p.c.f. set. This is replaced in the above, by the property $(\mathrm{H})$ and Proposition 2.5.

Proof. We let $\mathcal{F}_{j}$ be the family of all the cells $K_{\omega}$ with word length $|\omega|=j$. By Proposition 2.5, the IFS satisfies condition $(\mathrm{H})$, for our choice of $c$, we have $|x-y| \leq c \rho^{j}$ implies $x, y$ lie in the same cell or the neighboring $j$-cells. It follows that for $n>j$,

$$
\begin{aligned}
I_{n, j} & \leq \sum_{\substack{S, S^{\prime} \in \mathcal{F}_{j} \\
S \cap S^{\prime} \neq \emptyset}} \sum_{x \in S \cap V_{n}} \sum_{y \in S^{\prime} \cap V_{n}} \frac{1}{\left|V_{n}\right|^{2}}|u(x)-u(y)|^{2} \\
& \leq 2 \sum_{\substack{S, S^{\prime} \in \mathcal{F}_{j} \\
S \cap S^{\prime} \neq \emptyset}} \sum_{x \in S \cap V_{n}} \sum_{y \in S^{\prime} \cap V_{n}} \frac{1}{\left|V_{n}\right|^{2}}\left(|u(x)-u(z)|^{2}+|u(z)-u(y)|^{2}\right),
\end{aligned}
$$

where $z$ is any common vertex of $S$ and $S^{\prime}$. We observe the following estimates: (i) $\left|V_{n}\right| \asymp N^{n}$; (ii) $\#\left(S \cap V_{n}\right) \asymp N^{n-j}$; (iii) for each $z \in K_{\omega_{j}} \cap V_{j}, x \in K_{\omega_{n}} \cap V_{n}$, there exists a decreasing sequence of cells $\left\{K_{\omega_{k}}\right\}_{k=j}^{n}$ such that $\left|\omega_{k}\right|=k$ with $z \in K_{\omega_{j}} \cap V_{j}$, $x \in K_{\omega_{n}} \cap V_{n}$. Choose a sequence of vertices $\left\{z=x_{j}, x_{j+1}, \cdots, x_{n}=x\right\}$ such that $x_{k} \in K_{\omega_{k}} \cap V_{k}$ for $k=j, \cdots, n$. By Cauchy-Schwarz inequality, we have

$$
|u(z)-u(x)|^{2} \leq C_{1} \sum_{k=j}^{n-1} N^{k-j}\left|u\left(x_{k}\right)-u\left(x_{k+1}\right)\right|^{2} ;
$$

(iv) for each $S \in \mathcal{F}_{j}$, the cardinality of a fixed pair $(p, q):=\left(x_{k}, x_{k+1}\right)$ that appeared in $z \in S \cap V_{j}, x \in S \cap V_{n}$ is $\leq C^{\prime} N^{n-k}$ for some $C^{\prime}>0$ (counting all the possible successors of $k+1$ to $\left.x_{n}=x\right)$.

Now back to (3.3), by separating the sum there into two parts on $x$ and $y$ and adding separately, we obtain

$$
\begin{aligned}
& I_{n, j} \leq C_{2} N^{-(n+j)} \sum_{S \in \mathcal{F}_{j}}\left(\sum_{z \in S \cap V_{j}} \sum_{x \in S \cap V_{n}}|u(x)-u(z)|^{2}\right) \quad \text { (by (i)) } \\
& \leq C_{3} N^{-(n+j)} \sum_{S \in \mathcal{F}_{j}}\left(\sum_{k=j}^{n-1} \sum_{\substack{|\omega|=k, p, q \in K_{\omega} \cap V_{k+1} \\
K_{\omega} \subset S}} N^{k-j} \cdot N^{n-k}|u(p)-u(q)|^{2}\right) \quad \text { (by (ii)-(iv)) } \\
& =C_{3} \rho^{2 \alpha j} \sum_{k=j}^{n-1} \sum_{S \in \mathcal{F}_{j}} \sum_{\substack{|\omega|=k, p, q \in K_{\omega} \cap V_{k+1} \\
K_{\omega} \subseteq S}}|u(p)-u(q)|^{2} \quad\left(\text { by } N=\rho^{-\alpha}\right) .
\end{aligned}
$$

Finally, observe that for each pair $p, q \in K_{\omega} \cap V_{k+1}$ with $|\omega|=k$, any path (with no loop) connecting $p, q$ such that the two consecutive vertices belong to the same $K_{\tau} \cap V_{k+1},|\tau|=k+1$ has uniformly bounded length. Therefore by the triangle inequality,

$$
I_{n, j} \leq C_{4} \rho^{2 \alpha j} \sum_{k=j}^{n-1} E_{k+1}[u] \leq C_{4} \rho^{2 \alpha j} \sum_{k=j}^{n} E_{k}[u] .
$$

This completes the proof of the lemma.

Theorem 3.4. Let $K$ be a p.c.f. self-similar set. Then for $2 \sigma>\alpha$,

$$
[u]_{B_{2, \infty}^{\sigma}}^{2} \asymp \sup _{j \geq 0}\left\{\rho^{-(2 \sigma-\alpha) j} E_{j}[u]\right\} .
$$


Moreover, for any $\eta>\alpha / 2$, there is a uniform constant $C>0$ for all $\sigma>\eta$ so that the above "ऽ" holds.

Proof. It follows from Lemma 3.3 that

$$
\begin{aligned}
\rho^{-(2 \sigma+\alpha) j} I_{n, j} & \leq C \rho^{-(2 \sigma-\alpha) j} \sum_{k=j}^{n} \rho^{(2 \sigma-\alpha) k}\left(\sup _{k \geq 0} \rho^{-(2 \sigma-\alpha) k} E_{k}[u]\right) \\
& \leq C^{\prime} \sup _{k \geq 0} \rho^{-(2 \sigma-\alpha) k} E_{k}[u] .
\end{aligned}
$$

By letting $n \rightarrow \infty$, Lemma 3.2 implies the " $\leq$ " side of the theorem holds.

To prove the reverse inequality, we denote $I_{x, \omega}[u]:=\frac{1}{\mu(E)} \int_{K_{\omega}}|u(x)-u(y)|^{2} d \mu(y)$. For $p, q \in K_{\omega}$, using $|p-q|^{2} \leq 2\left(|p-x|^{2}+|x-q|^{2}\right), x \in K_{\omega}$, it is direct to show that

$$
E_{j}[u] \leq 2 \sum_{p, q \in V_{\omega},|\omega|=j}\left(I_{p, \omega}[u]+I_{q, \omega}[u]\right) \leq 4\left|V_{0}\right| \sum_{p \in V_{\omega},|\omega|=j} I_{p, \omega}[u] .
$$

For each $p \in V_{\omega}$ with $|\omega|=j$, there is a uniquely determined decreasing sequence of cells $\left\{K_{\omega_{k}}\right\}_{k=j}^{n}$ for $n$ sufficiently large, such that $p \in \bigcap_{k=j}^{n} K_{\omega_{k}}$ with $\omega_{j}=\omega$, $\left|\omega_{k+1}\right|=\left|\omega_{k}\right|+1$. Let $0<\delta<2 \sigma-\alpha$, by the Cauchy-Schwarz inequality, we have for $x_{k} \in K_{\omega_{k}}$,

$$
\begin{aligned}
& \left|u(p)-u\left(x_{j}\right)\right|^{2} \\
& \leq 2\left|u(p)-u\left(x_{n}\right)\right|^{2}+2\left(\sum_{k=j}^{n-1} \rho^{\delta(k-j)}\right)\left(\sum_{k=j}^{n-1} \rho^{-\delta(k-j)}\left|u\left(x_{k}\right)-u\left(x_{k+1}\right)\right|^{2}\right) .
\end{aligned}
$$

Since these $x_{k} \in K_{\omega_{k}}$ are arbitrary, the averages still hold:

$$
I_{p, \omega}[u] \leq I_{\omega_{n}}[u]+C \sum_{k=j}^{n-1} \rho^{-\delta(k-j)} \frac{1}{\mu\left(K_{\omega_{k}}\right)} \int_{K_{\omega_{k}}} I_{x, \omega_{k+1}}[u] d \mu(x) .
$$

We will make two estimations:

(i) For the first term on the right side of (3.7), we have $p, y \in K_{\omega_{n}}$, by Proposition 3.1,

$$
|u(p)-u(y)|^{2} \leq C|p-y|^{2 \sigma-\alpha}\|u\|_{B_{2, \infty}^{\sigma}}^{2} \leq C \rho^{(2 \sigma-\alpha) n}\|u\|_{B_{2, \infty}^{\sigma}}^{2}
$$

Note that the total number of $p$ in $V_{j}$ is $\asymp N^{j}=\rho^{-\alpha j}$. This implies that for $n>$ $\left(\frac{2 \sigma}{2 \sigma-\alpha}+1\right) j$.

$$
\rho^{-(2 \sigma-\alpha) j} \sum_{p \in V_{\omega},|\omega|=j} I_{\omega_{n}}[u] \leq C^{\prime} \rho^{(2 \sigma-\alpha)(n-j)} \rho^{-\alpha j}\|u\|_{B_{2, \infty}^{\sigma}}^{2}<C^{\prime}\|u\|_{B_{2, \infty}^{\sigma}}^{2},
$$


(ii) Combining (3.6) and the second term on the right side of (3.8), and observe that $|x-y| \leq \rho^{k}$ in the double integral, and $\mu\left(K_{\omega_{k}}\right) \asymp \mu\left(K_{\omega_{k+1}}\right) \asymp \rho^{k \alpha}$, we have

$$
\begin{aligned}
& \rho^{-(2 \sigma-\alpha) j} \sum_{p \in V_{\omega},|\omega|=j}\left(\sum_{k=j}^{n-1} \rho^{-\delta(k-j)} \frac{1}{\mu\left(K_{\omega_{k}}\right)} \int_{K_{\omega_{k}}} I_{x, \omega_{k+1}}[u] d \mu(x)\right) \\
& \leq C_{1} \rho^{-(2 \sigma-\alpha) j} \sum_{k=j}^{\infty} \rho^{-\delta(k-j)} \rho^{-2 \alpha k} \int_{K} \int_{B\left(x, \rho^{k}\right)}|u(x)-u(y)|^{2} d \mu(y) d \mu(x) \\
& \leq C_{2} \rho^{-(2 \sigma-\alpha) j} \sum_{k=j}^{\infty} \rho^{-\delta(k-j)} \rho^{-2 \alpha k}\left(\rho^{(2 \sigma+\alpha) k} \cdot\|u\|_{B_{2, \infty}^{\sigma}}^{2}\right) \\
& \leq C_{2} \sum_{k^{\prime}=0}^{\infty} \rho^{k^{\prime}((2 \sigma-\alpha)-\delta)} \cdot\|u\|_{B_{2, \infty}^{\sigma}}^{2} \leq C_{3}\|u\|_{B_{2, \infty}^{\sigma}}^{2} .
\end{aligned}
$$

The " $\geq$ " part of the theorem follows from (3.6)-(3.8) and the above two estimations.

For the last assertion, we inspect the constants in the above proof, the ones depend on $\sigma$ are those with $\sum_{n=0}^{\infty} \rho^{(2 \sigma-\alpha) n}<\infty$ (in (3.5) and (3.7)), hence for $\eta>$ $\alpha / 2$, there is a $C>0$ such that for all $2 \sigma \geq 2 \eta>\alpha$,

$$
C^{-1}[u]_{B_{2, \infty}^{\sigma}}^{2} \leq \sup _{j \geq 0}\left\{\rho^{-(2 \sigma-\alpha) j} E_{j}[u]\right\} \leq C[u]_{B_{2, \infty}^{\sigma}}^{2}, \quad u \in B_{2, \infty}^{\sigma} .
$$

It follows easily from Theorem 1.2 that

Corollary 3.5. For $2 \sigma>\alpha$, fix any integer $\ell \geq 1$, we have

$$
[u]_{B_{2, \infty}^{\sigma}}^{2} \asymp \sup _{n \geq 0}\left\{\rho^{-(2 \sigma-\alpha) n \ell} E_{n \ell}[u]\right\} .
$$

The following corollary is useful in the construction of functions in $B_{2, \infty}^{\sigma}$ on $K$ from a discrete form.

Corollary 3.6. Assume $2 \sigma>\alpha$, then for any function $u$ on $V_{*}$, if

$$
[u]_{B_{2, \infty}^{\sigma}\left(V_{*}\right)}^{2}:=\sup _{j \geq 0}\left\{\rho^{-(2 \sigma-\alpha) j} E_{j}[u]\right\}<\infty,
$$

then $u$ can be extended continuously to $\tilde{u}$ on $K$ with $\tilde{u} \in B_{2, \infty}^{\sigma}$.

Proof. The proof is based on the following inequality: there exists $C>0$ such that for any function $u$ on $V_{*}$, and any $x, y \in V_{*}$,

$$
|u(x)-u(y)|^{2} \leq C|x-y|^{2 \sigma-\alpha}[u]_{B_{2, \infty}^{\sigma}\left(V_{*}\right)}^{2} .
$$

(See also [23, Theorem 3.4].) Then $u$ is uniformly continuous on $V_{*}$ which is dense in $K$. Hence it has a continuous extension to $K$, and the extension is in $B_{2, \infty}^{\sigma}$ by Theorem 1.2.

We turn to prove (3.9). Given $x, y \in V_{*}$, let $k$ be the integer such that

$$
c_{0} \rho^{k+1} \leq|x-y|<c_{0} \rho^{k}
$$

where $c_{0}$ is the constant in condition $(\mathrm{H})$. Let $\omega$ and $\tau$ be two finite words such that $|\omega|=|\tau|=k$ and $x \in K_{\omega}, y \in K_{\tau}$. Observing that

$$
\operatorname{dist}\left(K_{\omega}, K_{\tau}\right) \leq|x-y|<c_{0} \rho^{k},
$$

and using condition $(\mathrm{H})$, we see that $K_{\omega} \cap K_{\tau} \neq \emptyset$. Let $z \in K_{\omega} \cap K_{\tau}$, we can find a decreasing sequence of cells starting with $\omega=\omega_{0}$ and a chain of points $x=$ 
$x_{0}, x_{1}, \cdots, x_{n}=z$ such that $x_{i} \in V_{\omega_{i}}$, and $\left\{V_{\omega_{i}}\right\}$ is a monotonic sequence (as in the proof of Lemma 3.3). Then

$$
\begin{aligned}
& |u(x)-u(z)| \leq \sum_{i=1}^{n}\left|u\left(x_{i-1}\right)-u\left(x_{i}\right)\right| \leq C \sum_{i=1}^{n}\left(\sum_{p, q \in V_{\omega},|\omega|=k+i}|u(p)-u(q)|^{2}\right)^{1 / 2} \\
& \leq C \sum_{i=1}^{n} \rho^{\frac{(2 \sigma-\alpha)(k+i)}{2}}[u]_{B_{2, \infty}^{\sigma}\left(V_{*}\right)} \leq C \rho^{\frac{k(2 \sigma-\alpha)}{2}}[u]_{B_{2, \infty}^{\sigma}\left(V_{*}\right)} .
\end{aligned}
$$

We get the same bound for $|u(y)-u(z)|$. Hence

$$
\begin{aligned}
|u(x)-u(y)| & \leq|u(x)-u(z)|+|u(y)-u(z)| \leq 2 C \rho^{\frac{k(2 \sigma-\alpha)}{2}}[u]_{B_{2, \infty}^{\sigma}\left(V_{*}\right)} \\
& \leq 2 C|x-y|^{\frac{2 \sigma-\alpha}{2}}[u]_{B_{2, \infty}^{\sigma}\left(V_{*}\right)}
\end{aligned}
$$

and proves (3.9).

\section{Discretizing $B_{2,2}^{\sigma}$}

The semi-norm $[u]_{B_{2,2}^{\sigma}}$ of $B_{2, \infty}^{\sigma}$ has the expression

$$
[u]_{B_{2,2}^{\sigma}}^{2}:=\int_{K} \int_{K} \frac{|u(x)-u(y)|^{2}}{|x-y|^{\alpha+2 \sigma}} d \mu(y) d \mu(x) .
$$

It is not hard to see that $[u]_{B_{2,2}^{\sigma}}^{2}$ is equivalent to

$$
\int_{0}^{1} \frac{d r}{r} \cdot \frac{1}{r^{\alpha+2 \sigma}} \int_{K} \int_{B(x, r)}(u(x)-u(y))^{2} d \mu(y) d \mu(x),
$$

as in (1.3), which can also be expressed as

$$
\sum_{j=0}^{\infty} \rho^{-(\alpha+2 \sigma) j} \int_{K} \int_{B\left(x, \rho^{j}\right)}(u(x)-u(y))^{2} d \mu(y) d \mu(x) .
$$

We have the following discretization of $[u]_{B_{2,2}^{\sigma}}$.

Proposition 4.1. Suppose $K$ is a p.c.f. self-similar set. Then for $2 \sigma>\alpha$,

$$
[u]_{B_{2,2}^{\sigma}}^{2} \asymp \sum_{j=0}^{\infty} \rho^{-(2 \sigma-\alpha) j} E_{j}[u] .
$$

Proof. In view of (4.2), $\|u\|_{B_{2, \infty}^{\sigma}} \leq\|u\|_{B_{2,2}^{\sigma}}$, hence by Proposition 3.1, $\iota: B_{2,2}^{\sigma} \rightarrow$ $C^{(2 \sigma-\sigma) / 2}(K)$ is a continuous embedding, so that every $u \in B_{2,2}^{\sigma}$ has a continuous representation.

By Lemma 3.3, we have

$$
\begin{aligned}
\sum_{j=0}^{\infty} \rho^{-(2 \sigma+\alpha) j} I_{j, n} & \leq C \sum_{j=0}^{\infty} \rho^{-(2 \sigma-\alpha) j} \sum_{k=j}^{\infty} \sum_{x, y \in V_{\omega},|\omega|=k}|u(x)-u(y)|^{2} \\
& \leq C^{\prime} \sum_{k=0}^{\infty} \rho^{-(2 \sigma-\alpha) k} \sum_{x, y \in V_{\omega},|\omega|=k}|u(x)-u(y)|^{2} .
\end{aligned}
$$

By using Fatou's lemma to pass the limit on $n$, the " $\leq$ " of (4.3) holds.

To prove the reverse inequality, we adopt the same estimation as (i) in the proof of Theorem 3.4 with some obvious adjustment. In (ii) we set the $\delta$ in (3.7) to 
be $(2 \sigma-\alpha) / 2$. Writing $I_{k}[u]=\int_{K} \int_{B\left(x, \rho^{k}\right)}|u(x)-u(y)|^{2} d \mu(y) d \mu(x)$, we have the estimate

$$
\begin{aligned}
& \sum_{j=0}^{n} \rho^{-(2 \sigma-\alpha) j} \sum_{p \in V_{\omega},|\omega|=j}\left(\sum_{k=j}^{n-1} \rho^{-\frac{(2 \sigma-\alpha)(k-j)}{2}} \frac{1}{\mu\left(K_{\omega_{k}}\right)} \int_{K_{\omega_{k}}} I_{x, \omega_{k+1}}[u] d \mu(x)\right) \\
& \leq C_{1} \sum_{j=0}^{n} \rho^{-(2 \sigma-\alpha) j} \sum_{k=j}^{n-1} \rho^{-\frac{(2 \sigma-\alpha)(k-j)}{2}} \rho^{-2 \alpha k} I_{k}[u] \leq C_{1} \sum_{k=0}^{\infty} \sum_{j=0}^{k} \rho^{\frac{(2 \sigma-\alpha)(k-j)}{2}} \cdot \rho^{-(2 \sigma+\alpha) k} I_{k}[u] \\
& \leq C_{2} \sum_{k=0}^{\infty} \rho^{-(2 \sigma+\alpha) k} I_{k}[u]=C_{2}\|u\|_{B_{2,2}^{\sigma}}^{2},
\end{aligned}
$$

and completes the proof of the reverse inequality.

\section{Convergence of $B_{2,2}^{\sigma}$ to $B_{2, \infty}^{\sigma}$}

In this section, we will make use of the $\Gamma$-convergence (Theorem 2.3) to prove the convergence of the Dirichlet forms on $B_{2,2}^{\sigma}$ to $B_{2, \infty}^{\sigma}$.

For $2 \sigma-\alpha>0$ and $u \in \ell\left(V_{n}\right)$ (or in $\ell\left(V_{*}\right)$ ), we write

$$
\mathcal{E}_{n}^{\sigma}[u]=\rho^{-(2 \sigma-\alpha) n} E_{n}[u],
$$

where $E_{n}[u]=\sum_{x, y \in V_{\omega},|\omega|=n}|u(x)-u(y)|^{2}$. It follows that for $\sigma, \sigma^{\prime}$,

$$
\mathcal{E}_{n}^{\sigma}[u]=\rho^{2\left(\sigma^{\prime}-\sigma\right) n} \cdot\left(\rho^{-\left(2 \sigma^{\prime}-\alpha\right) n} E_{n}[u]\right)=\rho^{2\left(\sigma^{\prime}-\sigma\right) n} \mathcal{E}_{n}^{\sigma^{\prime}}[u] .
$$

For $u_{0} \in \ell\left(V_{0}\right)$, we define the harmonic extension $u_{n} \in \ell\left(V_{n}\right), n \geq 1$ to be the unique one satisfies

$$
E_{n}\left[u_{n}\right]=\min \left\{E_{n}[v]: v \in \ell\left(V_{n}\right),\left.v\right|_{V_{0}}=u_{0}\right\} .
$$

For a sequence $\left\{n_{k}\right\}_{k=1}^{\infty}$, we can define inductively a sequence $u_{k}$ such that $E_{n_{k}}\left[u_{k}\right]=$ $\min \left\{E_{n_{k}}[v]: v \in \ell\left(V_{n_{k}}\right),\left.v\right|_{V_{n_{k-1}}}=u_{k-1}\right\}$. In this way for each $u_{0} \in \ell\left(V_{0}\right)$, we obtain a piecewise harmonic extension of $\widetilde{u} \in \ell\left(V_{*}\right)$. Also, for any $u \in \ell\left(V_{n}\right)$, we can consider $u$ restricted on each $n$-cells $V_{\omega}$, and use the above to extend $u$ to $\widetilde{u} \in \ell\left(V_{*}\right)$. Note that if $\sup _{n} \mathcal{E}_{n}^{\sigma}[\widetilde{u}]<\infty$, then $\widetilde{u} \in B_{2, \infty}^{\sigma}\left(V_{*}\right)$, and by Corollary 3.6, it can be extended to $K$ and belongs to $B_{2, \infty}^{\sigma}$.

In the following, we consider a more general type of extensions.

Definition 5.1. We say that a p.c.f. set $K$ satisfies property (E) if there exists $\sigma>0$ with $2 \sigma-\alpha>0$, and $C>0$ such that

(i) for any $u \in B_{2, \infty}^{\sigma}$ and for all $n \geq 1, E_{0}[u] \leq C \mathcal{E}_{n}^{\sigma}[u]$,

(ii) for any $u \in \ell\left(V_{0}\right)$, there exists an extension $\widetilde{u} \in B_{2, \infty}^{\sigma}$.

Remark 1. Let $\operatorname{Osc}(u, F)=\left|\max _{F} u-\min _{F} u\right|$ denote the oscillation of $u$ on $F$. Suppose $K$ satisfies (ii) of (E), we may require that $\operatorname{Osc}(\widetilde{u}, K)=\operatorname{Osc}\left(u, V_{0}\right)$, since if we set $v=(\widetilde{u} \vee \min u) \wedge \max u$, then $v$ is an extension of $u$ satisfying $E_{n}[v] \leq E_{n}[\widetilde{u}]$ for all $n$ and $\operatorname{Osc}(v, K)=\operatorname{Osc}\left(u, V_{0}\right)$.

Also, for any $u \in \ell\left(V_{m}\right)$, we can apply the above to each $K_{\omega},|\omega|=m$ piecewise, and obtain an extension $\widetilde{u} \in B_{2, \infty}^{\sigma} \operatorname{with} \operatorname{Osc}\left(\widetilde{u}, K_{\omega}\right)=\operatorname{Osc}\left(u, V_{\omega}\right)$ and $\mathcal{E}_{m}[u] \leq C \mathcal{E}_{n}^{\sigma}[\widetilde{u}]$ for all $n \geq m$.

Remark 2. Let $G_{n}$ denote the corresponding electrical network of $E_{n}[u]$. It is known [16, Theorem 2.1.6] that for any $n \geq 0$, there is an induced network of $G_{n}$ on 
$V_{0}$ with resistance $R_{n}(p, q)$ such that for $u \in \ell\left(V_{0}\right)$,

$$
\min \left\{E_{n}[v]: v \in \ell\left(V_{n}\right),\left.v\right|_{V_{0}}=u\right\}=\sum_{p, q \in V_{0}} \frac{1}{R_{n}(p, q)}|u(p)-u(q)|^{2} .
$$

In the well studied case that $R_{n}(p, q)=\lambda^{n}, \lambda>1$ (e.g., Sierpinski gasket, Vicsek cross), we have $\lambda E_{n+1}[u]=E_{n}[u]$, and $\lambda^{-1}$ is the renormalizing factor of the energy form. By taking piecewise harmonic extensions, and let $\sigma$ be such that $\rho^{-(2 \sigma-\alpha)}=\lambda$, and $C=1$, it is seen $K$ has property (E).

We will consider a sufficient condition for $K$ to have property (E) in the next section. The following proposition shows that the condition determines a unique $\sigma$, which is the critical exponent.

Proposition 5.2. Let $K$ be a p.c.f. self-similar set with property $(E)$ with respect to $\sigma$. Then $\sigma=\sigma^{*}$ necessarily, and $B_{2, \infty}^{\sigma^{*}}$ is dense in $C(K)$ (in the supremum norm).

Proof. We first show that $B_{2, \infty}^{\sigma}$ is dense in $C(K)$. Let $v \in C(K)$, and let $v_{n}=\left.v\right|_{V_{n}}$. By property (E)(ii), we have piecewise extensions $\left\{\widetilde{v}_{n}\right\}_{n} \subset B_{2, \infty}^{\sigma}$ (as in Remark 1) and they are continuous (Proposition 3.1). For $\varepsilon>0$, we can find $m$ such that $\operatorname{Osc}\left(v, K_{\omega}\right) \leq \varepsilon$ for all $\omega$ with $|\omega|=m$, hence $\operatorname{Osc}\left(\widetilde{v}_{m}, K_{\omega}\right) \leq \varepsilon$. It follows that for any $x \in K_{\omega},|\omega|=m$, and for any fixed $q \in K_{\omega} \cap V_{m}$, we have

$$
\left|\widetilde{v}_{m}(x)-v(x)\right| \leq\left|\widetilde{v}_{m}(x)-\widetilde{v}_{m}(q)\right|+\left|\widetilde{v}_{m}(q)-v(q)\right|+|v(q)-v(x)| \leq 2 \varepsilon .
$$

This shows that $B_{2, \infty}^{\sigma}$ is dense in $C(K)$.

Next observe that $B_{2,2}^{\sigma} \subset B_{2, \infty}^{\sigma} \subset B_{2,2}^{\sigma-\varepsilon}$ for any small $\varepsilon>0$. This implies $\sigma^{*}$ can be defined in terms of $B_{2,2}^{\sigma}$, i.e.,

$$
\sigma^{*}=\sup \left\{\sigma>0: B_{2,2}^{\sigma} \cap C(K) \text { is dense in } C(K)\right\} .
$$

For each nonconstant $u \in B_{2, \infty}^{\sigma}$, by considering subcells if necessary, we may assume that $E_{0}[u]>0$. By applying property (E)(i) to $u$, we see that for any $n>0$,

$$
\mathcal{E}_{n}^{\sigma}[u] \geq C^{-1} E_{0}[u] .
$$

This implies $[u]_{B_{2,2}^{\sigma}}^{2}=\sum_{n=0}^{\infty} \mathcal{E}_{n}^{\sigma}[u]=\infty$, and $u \notin B_{2,2}^{\sigma}$. Hence we have $B_{2,2}^{\sigma}=$ \{const. function\}. This implies that for any $\sigma^{\prime}>\sigma, B_{2, \infty}^{\sigma^{\prime}}$ is trivial, in particular, not dense in $C(K)$. Therefore $\sigma=\sigma^{*}$.

We write $\mathcal{E}_{2,2}^{\sigma}$ to be the Dirichlet form defined by $\sum_{j=0}^{\infty} \mathcal{E}_{j}^{\sigma}[u]=\sum_{j=0}^{\infty} \rho^{-(2 \sigma-\alpha) j} E_{j}[u]$, then $\mathcal{E}_{2,2}^{\sigma}$ has domain $B_{2,2}^{\sigma}$. Our main theorem is

Theorem 5.3. Suppose $K$ is a p.c.f. self-similar set and has property (E). Then $K$ admits a local regular self-similar Dirichlet form $\left(\mathcal{E}^{\sigma^{*}}, \mathcal{F}\right)$ with domain $\mathcal{F}=B_{2, \infty}^{\sigma^{*}}$, and there exists $C>0$ such that for $u \in \mathcal{F}$,

$$
C^{-1} \mathcal{E}^{\sigma^{*}}[u] \leq \lim _{\sigma \uparrow \sigma^{*}}\left(\sigma^{*}-\sigma\right) \mathcal{E}_{2,2}^{\sigma}[u] \leq \varlimsup_{\sigma \uparrow \sigma^{*}}\left(\sigma^{*}-\sigma\right) \mathcal{E}_{2,2}^{\sigma}[u] \leq C \mathcal{E}^{\sigma^{*}}[u] .
$$

Proof. Consider the family of closed forms $\left\{\left(\sigma^{*}-\sigma\right) \mathcal{E}_{2,2}^{\sigma}: \sigma<\sigma^{*}, 2 \sigma-\alpha>0\right\}$, there is a sequence $\sigma_{n} \nearrow \sigma^{*}$ and $\left(\sigma^{*}-\sigma_{n}\right) \mathcal{E}_{2,2}^{\sigma_{n}} \Gamma$-converges to some closed form $\mathcal{E}^{*}$ in $L^{2}(K, \mu)$ in the wide sense (Theorem 2.3). Let $\mathcal{F}=\left\{u \in L^{2}(K, \mu): \mathcal{E}^{*}[u]<\infty\right\}$.

We observe that for $u \in B_{2, \infty}^{\sigma^{*}}$. Taking $u_{n}=u$, by Definition 2.2(i) and (5.1), we have

$$
\mathcal{E}^{*}[u] \leq \varliminf_{n \rightarrow \infty}\left(\sigma^{*}-\sigma_{n}\right) \mathcal{E}_{2,2}^{\sigma_{n}}[u]=\varliminf_{n \rightarrow \infty}\left(\sigma^{*}-\sigma_{n}\right) \sum_{j=0}^{\infty} \rho^{2\left(\sigma^{*}-\sigma_{n}\right) j} \mathcal{E}_{j}^{\sigma^{*}}[u] \leq C_{1} \sup _{j \geq 0} \mathcal{E}_{j}^{\sigma^{*}}[u] .
$$


Therefore we have $\mathcal{E}^{*}[u] \leq C^{\prime}[u]_{B_{2, \infty}^{\sigma^{*}}}^{2}$, so that $B_{2, \infty}^{\sigma^{*}} \subseteq \mathcal{F}$.

On the other hand, assume that $u \in \mathcal{F}$. By the $\Gamma$-convergence (Definition 2.2(ii)), for any $u \in L^{2}(K, \mu)$, there exists $\left\{u_{n}\right\}_{n} \subset L^{2}(K, \mu)$ converges to $u$ strongly such that

$$
\varlimsup_{n \rightarrow \infty}\left(\sigma^{*}-\sigma_{n}\right) \mathcal{E}_{2,2}^{\sigma_{n}}\left[u_{n}\right] \leq \mathcal{E}^{*}[u]<\infty .
$$

Hence there is some $N_{0}$ such that for $n \geq N_{0}$, (the case $\mathcal{E}^{*}[u]=0$ is similar)

$$
\left(\sigma^{*}-\sigma_{n}\right) \mathcal{E}_{2,2}^{\sigma_{n}}\left[u_{n}\right] \leq 2 \mathcal{E}^{*}[u],
$$

which implies $u_{n} \in B_{2,2}^{\sigma_{n}}$ for $n \geq N_{0}$ (Proposition 4.1). As $B_{2,2}^{\sigma_{n}} \subset C(K), u_{n}$ is pointwise defined. Hence that $\mathcal{E}_{j}^{\sigma^{*}}\left[u_{n}\right]$ is well defined. By (5.1), we have

$$
\begin{aligned}
2 \mathcal{E}^{*}[u] & \geq\left(\sigma^{*}-\sigma_{n}\right) \sum_{j=0}^{\infty} \rho^{2\left(\sigma^{*}-\sigma_{n}\right) j} \mathcal{E}_{j}^{\sigma^{*}}\left[u_{n}\right] \\
& \geq\left(\sigma^{*}-\sigma_{n}\right) \sum_{j=m}^{\infty} \rho^{2\left(\sigma^{*}-\sigma_{n}\right) j} \mathcal{E}_{j}^{\sigma^{*}}\left[u_{n}\right] \\
& \left.\geq \frac{1}{C}\left(\sigma^{*}-\sigma_{n}\right) \sum_{j=m}^{\infty} \rho^{2\left(\sigma^{*}-\sigma_{n}\right) j} \mathcal{E}_{m}^{\sigma^{*}}\left[u_{n}\right] \quad \text { (by property }(\mathrm{E})(\mathrm{i})\right) \\
& \geq \frac{1}{C_{2}} \rho^{2\left(\sigma^{*}-\sigma_{n}\right) m} \mathcal{E}_{m}^{\sigma^{*}}\left[u_{n}\right]=\frac{1}{C_{2}} \mathcal{E}_{m}^{\sigma_{n}}\left[u_{n}\right] .
\end{aligned}
$$

From this and the second part of Theorem 1.2, there is a constant $C^{\prime \prime}>0$ such that for $n \geq N_{0}$,

$$
\left[u_{n}\right]_{B_{2, \infty}^{\sigma_{n}}}^{2} \leq C^{\prime \prime} \sup _{m \geq 0} \mathcal{E}_{m}^{\sigma_{n}}\left[u_{n}\right] \leq C_{3} \mathcal{E}^{*}[u],
$$

where $C_{3}=2 C^{\prime \prime} C_{2}$. By the imbedding $B_{2, \infty}^{\sigma_{N_{0}}} \hookrightarrow C^{\left(2 \sigma_{N_{0}}-\alpha\right) / 2}$, we see that $\left\{u_{n}\right\}_{n \geq N_{0}}$ are equi-continuous, and furthermore by $u_{n} \rightarrow u$ in $L^{2}(K, \mu)$, we have $\left\{u_{n}\right\}_{n \geq N_{0}}$ are uniformly bounded. Hence $\left\{u_{n}\right\}_{n}$ is compact in $C(K)$, and there is a subsequence $\left\{u_{n_{k}}\right\}_{k \geq 0}$ and some $v \in C(K)$ such that $u_{n_{k}} \rightarrow v$ in $C(K)$. Therefore $u_{n_{k}} \rightarrow v$ in $L^{2}(K, \mu)$, which yields $v=u$. Hence $u_{n_{k}} \rightarrow u$ uniformly on $K$.

It follows that $\lim _{k \rightarrow \infty} E_{m}\left[u_{n_{k}}\right]=E_{m}[u]$ for any $m \geq 0$. In view of (5.2), we see that $\mathcal{E}_{m}^{\sigma_{n_{k}}}\left[u_{n_{k}}\right] \leq 2 C_{2} \mathcal{E}^{*}[u]$, and by letting $k \rightarrow \infty$, and take supremum on $m$, we have

$$
\sup _{m \geq 0} \mathcal{E}_{m}^{\sigma^{*}}[u] \leq 2 C_{2} \mathcal{E}^{*}[u] \text {. }
$$

This gives $[u]_{B_{2, \infty}^{\sigma^{*}}}^{2} \leq 2 C^{\prime \prime} C_{2} \mathcal{E}^{*}[u]$, so that $\mathcal{F} \subseteq B_{2, \infty}^{\sigma^{*}}$. Together with the first part, we have $\mathcal{F}=B_{2, \infty}^{\sigma^{*}}$. By Proposition 5.2, $\mathcal{F}$ is dense in $C(K)$.

Next, we use a standard argument (see [18], [10]) to construct a closed form $\left(\mathcal{E}^{\sigma^{*}}, \mathcal{F}\right)$ that is self-similar and equivalent to $\left(\mathcal{E}^{*}, \mathcal{F}\right)$. We write $\lambda=\rho^{-\left(2 \sigma^{*}-\alpha\right)}$. For $k \geq 0$ and $u \in \mathcal{F}$, let

$$
\overline{\mathcal{E}}^{(k)}[u]=\lambda^{k} \sum_{|\omega|=k} \mathcal{E}^{*}\left[u \circ F_{\omega}\right],
$$

and for $m \geq 1$, define $\widetilde{\mathcal{E}}^{(m)}[u]=\frac{1}{m} \sum_{k=0}^{m-1} \overline{\mathcal{E}}^{(k)}[u]$. Then for all $u \in \mathcal{F}$, we have $\widetilde{\mathcal{E}}^{(m)}[u] \asymp[u]_{B_{2, \infty}^{\sigma^{*}}}^{2}$. By a diagonal argument, we can find a subsequence, which we still 
denote by $\widetilde{\mathcal{E}}^{(m)}$ such that $\left\{\widetilde{\mathcal{E}}^{(m)}[u]\right\}_{m}$ converges for all $u \in \mathcal{F}$. We denote by

$$
\mathcal{E}^{\sigma^{*}}[u]=\lim _{m \rightarrow \infty} \widetilde{\mathcal{E}}^{(m)}[u], \quad u \in \mathcal{F} .
$$

It is clear that $\left(\mathcal{E}^{\sigma^{*}}, \mathcal{F}\right)$ is still a closed form.

By (5.3), we have $\overline{\mathcal{E}}^{(k+1)}[u]=\lambda \sum_{i=1}^{N} \overline{\mathcal{E}}^{(k)}\left[u \circ F_{i}\right]$, which, by taking limit, shows that

$$
\mathcal{E}^{\sigma^{*}}[u]=\lambda \sum_{i=1}^{N} \mathcal{E}^{\sigma^{*}}\left[u \circ F_{i}\right], \quad u \in \mathcal{F},
$$

and the self-similar identity of $\left(\mathcal{E}^{\sigma^{*}}, \mathcal{F}\right)$ follows. We also have that

$$
\mathcal{E}^{\sigma^{*}}[u] \asymp \mathcal{E}^{*}[u] \asymp[u]_{B_{2, \infty}^{\sigma^{*}}}^{2}, \quad u \in \mathcal{F} .
$$

The Markovian property of $\mathcal{E}^{\sigma^{*}}$ is clear (e.g., [20, Theorem 2.8.1]), and the locality of $\mathcal{E}^{\sigma^{*}}$ is an immediate consequence of self-similar identity (see the proof in [16, Theorem 3.4.6]). Hence $\left(\mathcal{E}^{\sigma^{*}}, \mathcal{F}\right)$ is a local regular Dirichlet form.

\section{A sufficient condition}

In the following, we give a sufficient condition for property (E) to hold.

Theorem 6.1. Let $K$ be a p.c.f. self-similar set. Assume that there exists $\lambda>1$ and $C>0$ such that for any two distinct points $p, q \in V_{0}$, and for any $n \geq 1$,

$$
C^{-1} \lambda^{n} \leq R_{n}(p, q) \leq C \lambda^{n} .
$$

Then $K$ satisfies property $(E)$.

The idea of proof is based on a uniform Harnack inequality for the nonnegative harmonic functions (Proposition 6.3), similar to that used in [10] dealing with the Sierpinski carpet. We will need a few lemmas.

For $m \geq 1$, for $x \in V_{m}$, we denote

$$
\Omega_{m}(x):=\bigcup\left\{K_{\omega}:|\omega|=m, x \in K_{\omega}\right\}, \quad \mathcal{L}_{m}(x)=\left\{\omega:|\omega|=m, K_{\omega} \subseteq \Omega_{m}(x)\right\},
$$

also we let $\Omega_{m}^{\prime}(x):=\bigcup\left\{K_{\omega}:|\omega|=m, K_{\omega} \cap \Omega_{m}(x) \neq \emptyset\right\}$.

Lemma 6.2. Suppose $K$ satisfies (6.1). Then there exists $C_{0}>0$ such that for any $x \in V_{m}$, and any nonnegative harmonic function $u$ on $\Omega_{m}^{\prime}(x) \cap V_{n}, n \geq m$, we have

$$
u\left(F_{\omega}(p)\right) \leq C_{0} u\left(F_{\omega}(q)\right) \quad \text { for any } \omega \in \mathcal{L}_{m}(x), \quad p, q \in V_{0} .
$$

Consequently, there is $C_{1}>0$ (independent of $m, n$ and $u$ ) such that

$$
\max _{\Omega_{m}(x) \cap V_{n}} u \leq C_{1} \min _{\Omega_{m}(x) \cap V_{n}} u \text {. }
$$

Proof. Fix a pair $p, q \in V_{0}, p \neq q$. Without loss of generality, we assume that $u\left(F_{\omega}(p)\right)=1$. Clearly, we have $\Omega_{m}\left(F_{\omega}(q)\right) \subseteq \Omega_{m}^{\prime}(x)$, so $u$ is nonnegative and harmonic on $\Omega_{m}\left(F_{\omega}(q)\right)$, and $u$ is uniquely determined by the values of $u$ on $\bigcup_{\eta \in \mathcal{L}_{m}}\left\{F_{\eta}\left(V_{0}\right)\right\} \backslash\left\{F_{\omega}(q)\right\}$, where $\mathcal{L}_{m}:=\mathcal{L}_{m}\left(F_{\omega}(q)\right)$.

Define $v$ on $V_{m}$ with 0 outside $\left\{F_{\omega}(p), F_{\omega}(q)\right\}, 1$ on $F_{\omega}(p)$ and harmonic at $F_{\omega}(q)$. It is clear that $u\left(F_{\omega}(q)\right) \geq v\left(F_{\omega}(q)\right)$. Let $\widetilde{v}$ be the harmonic extension of $v$ on $V_{n}$. 
Write $\gamma=v \circ F_{\omega}(q)$, then we have

$$
\begin{aligned}
E_{n}[\widetilde{v}]= & \sum_{\eta \in \mathcal{L}_{m}} E_{n-m}\left[v \circ F_{\eta}\right]=\sum_{\eta \in \mathcal{L}_{m}} \sum_{x, y \in V_{0}} \frac{1}{R_{n-m}(x, y)}\left(v \circ F_{\eta}(x)-v \circ F_{\eta}(y)\right)^{2} \\
& =\frac{(1-\gamma)^{2}}{R_{n-m}(p, q)}+\sum_{\eta \in \mathcal{L}_{m}} \sum_{x \in V_{0}, x \neq p, q} \frac{\gamma^{2}}{R_{n-m}(x, q)}+\sum_{\eta \in \mathcal{L}_{m}} \sum_{x \in V_{0}, x \neq p, q} \frac{1}{R_{n-m}(x, p)} \\
& =: \mu_{1}(1-\gamma)^{2}+\mu_{2} \gamma^{2}+\mu_{3} .
\end{aligned}
$$

Since $v$ is harmonic on $F_{\omega}(q)$ with boundary $V_{m} \backslash F_{\omega}(q)$, the above energy expression has minimum for $v\left(F_{\omega}(q)\right):=\gamma=\frac{\mu_{1}}{\mu_{1}+\mu_{2}}$. Now observe that $C^{-1} \lambda^{m-n} \leq R_{n-m}(x, y) \leq$ $C \lambda^{m-n}$ for $x, y \in V_{0}$ by assumption, and that $\#\left\{(\eta, x): \eta \in \mathcal{L}_{m}, x \in V_{0}\right\}$ is uniformly bounded. Hence there exists $c_{0}$ independent of $m, n, \omega, u$ such that $v\left(F_{\omega}(q)\right) \geq c_{0}>0$. By taking $C_{0}=c_{0}^{-1}$, we have

$$
u\left(F_{\omega}(p)\right)=1 \leq C_{0} v\left(F_{\omega}(q)\right) \leq C_{0} u\left(F_{\omega}(q)\right),
$$

and completes the proof of the first part.

By the maximum principle of the harmonic function $u$ on $\Omega_{m}(x), u$ takes maximum and minimum on the set $\bigcup_{\omega \in \mathcal{L}_{m}(x)}\left\{F_{\omega}\left(V_{0}\right)\right\} \backslash\{x\}$. On the other hand, applying the above result to these $K_{\omega}$ for $\omega \in \mathcal{L}_{m}(x)$, it follows that for any $y \in$ $\bigcup_{\omega \in \mathcal{L}_{m}(x)}\left\{F_{\omega}\left(V_{0}\right)\right\}$

$$
C_{0}^{-1} u(y) \leq u(x) \leq C_{0} u(y)
$$

This implies that

$$
\max _{\Omega_{m}(x) \cap V_{n}} u \leq C_{0}^{2} \min _{\Omega_{m}(x) \cap V_{n}} u .
$$

Proposition 6.3. There is $C>0$ and $\delta \in(0,1)$ such that for any nonnegative harmonic function $u$ on $B(x, r) \cap V_{n}$, where $x \in K$, and $3 a \rho^{n}<r<1(a=\operatorname{diam}(K))$,

$$
\max _{B(x, \delta r) \cap V_{n}} u \leq C \min _{B(x, \delta r) \cap V_{n}} u, \quad \text { uniform for } n \geq 0 .
$$

Proof. Let $m$ be the smallest integer such that $3 a \rho^{m}<r$, then $m \leq n$. We pick $|\omega|=m$ such that $x \in K_{\omega}$. Then for any $p \in F_{\omega}\left(V_{0}\right)$, we have $\Omega_{m}^{\prime}(p) \subset B(x, r)$. Hence $u$ is nonnegative and harmonic on $\Omega_{m}^{\prime}(p)$. By Lemma 6.2, we have

$$
\max _{\Omega_{m}(p) \cap V_{n}} u \leq C_{0} \min _{\Omega_{m}(p) \cap V_{n}} u, \quad p \in F_{\omega}\left(V_{0}\right) .
$$

Denote $\Omega(x)=\left\{K_{\tau}:|\tau|=m, K_{\tau} \cap K_{\omega} \neq \emptyset\right\}\left(\subset \Omega_{m}^{\prime}(p)\right)$. Since $\max _{\Omega(x) \cap V_{n}} u$ and $\min _{\Omega(x) \cap V_{n}} u$ take place at those $F_{\tau}\left(V_{0}\right)$, by using (6.2) twice, we have

$$
\max _{\Omega(x) \cap V_{n}} u \leq C_{0}^{2} \min _{\Omega(x) \cap V_{n}} u \text {. }
$$

On the other hand, $K$ satisfies condition $(\mathrm{H})$ (Proposition 2.5), which yields a $0<$ $\delta<1$ such that $B(x, \delta r) \subseteq \Omega(x)$. Hence our assertion follows.

In the following, we use Proposition 6.3 and an argument in [1] to obtain the uniform Hölder continuity of bounded harmonic functions away from the boundary.

Lemma 6.4. For small $\eta>0$, let $K_{\eta}:=K \backslash\left(\bigcup_{p \in V_{0}} B(p, \eta)\right)(\neq \emptyset)$. Then there exists $C_{\eta}>0$ such that for any $n \geq 1$ and $u$ a function on $V_{n}$ harmonic on $V_{n} \backslash V_{0}$, we have

$$
|u(x)-u(y)| \leq C_{\eta}|x-y|^{\gamma} \max _{V_{n}}|u|, \quad \forall x, y \in K_{\eta} \cap V_{n},
$$

where $\gamma>0$ is independent of $\eta, n$ and $u$. 
Proof. We apply the argument as in [1, Theorem 3.9]. To do this, we first obtain the oscillation inequality for harmonic functions by the uniform Harnack inequality.

By using Proposition 6.3 and an argument of Moser [21], we obtain $0<\varepsilon, \delta<1$ such that for any $n \geq m \geq 1$, any harmonic function $u$ on $B \cap V_{n}$, with $x \in K$ and $3 a \rho^{n}<r<1$, we have

$$
\operatorname{Osc}\left(u, \delta B \cap V_{n}\right) \leq \varepsilon \cdot \operatorname{Osc}\left(u, B \cap V_{n}\right),
$$

where $B=B(x, r)$ and $\delta B=B(x, \delta r)$.

For $x, y \in K_{\eta} \cap V_{n}$, let $k$ be the integer such that $\delta^{k+1} \leq|x-y|<\delta^{k}$. Assume $m$ is the integer such that $\delta^{m} \leq \eta<\delta^{m-1}$. If $k \geq m$, we have

$$
\begin{aligned}
|u(x)-u(y)| & \leq \operatorname{Osc}\left(u, B\left(x, \delta^{k}\right)\right) \leq \varepsilon \operatorname{Osc}\left(u, B\left(x, \delta^{k-1}\right)\right) \\
& \vdots \\
& \leq \varepsilon^{k-m} \operatorname{Osc}\left(u, B\left(x, \delta^{m}\right)\right) \\
& \leq C \eta^{-\log \varepsilon / \log \delta}|x-y|^{\log \varepsilon / \log \delta} \max _{V_{n}}|u| .
\end{aligned}
$$

If $k \leq m$, then $|x-y| \geq \delta^{2} \eta$, we have

$$
|u(x)-u(y)| \leq 2 \max _{V_{n}}|u| \leq 2\left(\delta^{2} \eta\right)^{-1}|x-y| \max _{V_{n}}|u| .
$$

Combining the above two cases, our assertion holds.

Proof of Theorem 6.1. Let $\lambda=\rho^{-(2 \sigma-\alpha)}$. As $\lambda>1$, it follows that $2 \sigma-\alpha>$ 0 . That $K$ satisfies Property (E)(i) is simple because using $R_{n}(p, q) \asymp \lambda^{n}$ (see Remark 2), we have for any $u \in B_{2, \infty}^{\sigma}$, and $v$ the harmonic extension of $\left.u\right|_{V_{0}}$ on $V_{n}$,

$$
E_{0}[u] \leq C \lambda^{n} E_{n}[v] \leq C \lambda^{n} E_{n}[u]=C \mathcal{E}_{n}^{\sigma}[u] .
$$

We prove $K$ satisfies property (E)(ii). Let $u \in \ell\left(V_{0}\right)$ with values $u(p)$ for $p \in V_{0}$ and $u_{n}$ be the harmonic function on $V_{n}$ with $\left.u_{n}\right|_{V_{0}}=u$. By Lemma 6.4, we see that for any $\eta>0$, let $K_{\eta}:=K \backslash \bigcup_{p \in V_{0}} B(p, \eta)$, we have

$$
\left|u_{n}(x)-u_{n}(y)\right| \leq C_{\eta}|x-y|^{\gamma} \max _{V_{0}}|u| \quad \forall x, y \in K_{\eta} \cap V_{n} .
$$

By using the well-known Whitney extension and trace theorem on closed sets [15, $\S 2.2$, Theorem 2], we can extend $u_{n}$ from $K_{\eta} \cap V_{n}$ to be $\widetilde{u}_{n}$ on $\mathbf{R}^{d}$, then restrict back on $K$ preserving the bound $C_{\eta}$ and the Hölder exponent. This together with the uniform boundedness of $\left\{\widetilde{u}_{n}\right\}_{n \geq 0}$ yield the compactness of $\left\{\widetilde{u}_{n}\right\}_{n \geq 0}$ in $C\left(K_{\eta}\right)$. Therefore there is a subsequence $\left\{\widetilde{u}_{n_{k}}\right\}_{k \geq 0}$ and $\widetilde{u}$ on $K_{\eta}$ such that $\widetilde{u}_{n_{k}} \rightarrow \widetilde{u}$ uniformly on $K_{\eta}$. Next by letting $\eta \rightarrow 0$, and by a diagonal argument, we obtain a subsequence, which we still denote by $\left\{\widetilde{u}_{n_{k}}\right\}_{k \geq 0}$, and a continuous $\widetilde{u}$ on $K \backslash V_{0}$ such that $\widetilde{u}_{n_{k}} \rightarrow \widetilde{u}$ pointwise on $K \backslash V_{0}$.

To see the continuity of $\widetilde{u}$ on the boundary points $p \in V_{0}$, we first claim $\widetilde{u}_{n}(z), z \in$ $K$ converges to $u_{n}(p)$ uniformly on $n$ as $z \rightarrow p$. Indeed, let $m \geq 1$ be fixed and $K_{\omega}$ be an $m$-cell contains $p$, assume $n \geq m$, then we have

$$
\begin{aligned}
E_{n}\left[u_{n}\right] & \geq \sum_{x, y \in V_{0}} \frac{1}{R_{n-m}(x, y)}\left(u_{n} \circ F_{\omega}(x)-u_{n} \circ F_{\omega}(y)\right)^{2} \\
& \geq C^{-1} \lambda^{m-n} \sum_{x, y \in V_{0}}\left(u_{n} \circ F_{\omega}(x)-u_{n} \circ F_{\omega}(y)\right)^{2},
\end{aligned}
$$


Also, as $u_{n}$ is the harmonic extension of $u \in \ell\left(V_{0}\right)$, we have by (6.1) that for any $n \geq 0$,

$$
E_{n}\left[u_{n}\right]=\sum_{p, q \in V_{0}} R_{n}(p, q)^{-1}\left(u_{n}(p)-u_{n}(q)\right)^{2} \leq C \lambda^{-n} E_{0}[u] .
$$

Hence $\left|u_{n}(x)-u_{n}(p)\right|^{2} \leq C \lambda^{-m}$ for $x, p \in F_{\omega}\left(V_{0}\right),|\omega|=m, m>0$. The property $(\mathrm{H})$ on $K$ implies the estimate also holds for small ball with center at $p$ (same argument as the last part of proof in Proposition 6.3), and the claim follows. Therefore by setting $\widetilde{u}$ with boundary values $u(p)$ for $p \in V_{0}$, we obtain $\widetilde{u} \in C(K)$.

We show that $\widetilde{u}$ is the required function in property (E)(ii). We fix $m \geq 0$. As $u_{n_{k}} \rightarrow \widetilde{u}$ uniformly on any $K_{\eta}$, we have (as finite sums)

$$
\mathcal{E}_{m}^{\sigma^{*}}[\widetilde{u}]=\lim _{k \rightarrow \infty} \lambda^{m} E_{m}\left[u_{n_{k}}\right] .
$$

We claim that

$$
\lambda^{m} E_{m}\left[u_{n_{k}}\right] \leq C^{2} E_{0}[u] .
$$

Indeed for any $n_{k} \geq m$, by (6.5), we have $E_{n_{k}}\left[u_{n_{k}}\right] \leq C \lambda^{-n_{k}} E_{0}[u]$. Also by the harmonicity of $u_{n_{k}}$ on each $\left(n_{k}-m\right)$-cell, we have

$$
\begin{aligned}
E_{n_{k}}\left[u_{n_{k}}\right] & =\sum_{|\omega|=m} \sum_{p, q \in V_{0}} R_{n_{k}-m}(p, q)^{-1}\left(u_{n_{k}}\left(F_{\omega}(p)\right)-u_{n_{k}}\left(F_{\omega}(q)\right)\right)^{2} \\
& \geq C^{-1} \lambda^{-\left(n_{k}-m\right)} E_{m}\left[u_{n_{k}}\right] .
\end{aligned}
$$

The claims follows from these two estimates. Substituting this into (6.6), and observing that $C$ does not depend on $m$, we have $\sup _{m \geq 0} \mathcal{E}_{m}^{\sigma^{*}}[\widetilde{u}] \leq C^{2} E_{0}[u]$, This implies $\widetilde{u} \in B_{2, \infty}^{\sigma^{*}}$ (Corollary 3.6), and completes the proof.

Remark. For the condition $C^{-1} \lambda^{n} \leq R_{n}(p, q) \leq C \lambda^{n}, p, q \in V_{0}, n \geq 0$, we can relax the upper bound slightly (keep the lower bound): for any $p, q \in V_{0}$, there is some chain $p=p_{1}, p_{2}, \cdots, p_{k}=q$ in $V_{0}$ such that

$$
R_{n}\left(p_{i}, p_{i+1}\right) \leq C \lambda^{n}, \quad \text { for } i=1 \cdots, k-1,
$$

and all the above proofs go through. Indeed the only change is in showing $u\left(F_{\omega}(p)\right) \leq$ $C_{0} u\left(F_{\omega}(q)\right)$ in Theorem 6.1. We apply the same argument to each pair $\left(p_{i}, p_{i+1}\right)$, then

$$
u\left(F_{\omega}(p)\right) \leq C_{0} u\left(F_{w}\left(p_{1}\right)\right) \leq \cdots \leq C_{0}^{k} u\left(F_{\omega}(q)\right) .
$$

Hence Theorem 6.1 holds with this new sufficient condition.

\section{Examples and remarks}

For the Sierpinski gasket $K$, the boundary is the three vertices $V_{0}=\left\{p_{i}\right\}_{i=1}^{3}$. It is well known that the trace of the $n$-network on $V_{0}$ satisfying $R_{n}\left(p_{i}, p_{j}\right)=\left(\frac{5}{3}\right)^{n}$ for $i \neq j$ [16]. From $\frac{5}{3}=\rho^{-\left(2 \sigma^{*}-\alpha\right)}$ with $\rho=\frac{1}{2}$ and $\alpha=\frac{\log 3}{\log 2}$, we see that the critical exponent is $\sigma^{*}=\frac{\log 5}{2 \log 2}$ [14]. It follows that $K$ has property (E) (Theorem 6.1) and the convergence of the non-local Dirichlet forms on $B_{2,2}^{\sigma}$ to the local Dirichlet form on $B_{2, \infty}^{\sigma^{*}}$ holds (Theorem 5.3). By the uniqueness of the self-similar Dirichlet form on $K$ [24], we see that the Dirichlet form obtained in this way is the canonical one on $K$. We remark that in [29], Yang first used the $\Gamma$-convergence to prove this convergence theorem on the Sierpinski gasket. The present approach is a modification of his technique, it greatly simplified and generalized his result. This convergence theorem also follows from another probabilistic approach of Pietruska-Pałuba on some simple nested fractals under the assumption that sub-Gaussian heat kernel exists. 
In [11], we use the Sierpinski-type self-similar sets to study the critical exponents of the Besov space, they also provide interesting examples and insights for the convergence of the Dirichlet forms. Let $K_{1}$ be the self-similar set with 15 similitudes on a triangle with vertices $V_{0}=\left\{p_{i}\right\}_{i=1}^{3}$, and with contraction ratio $\rho=\frac{1}{7}$ as in Figure 1 . $K_{1}$ has Hausdorff dimension $\frac{\log 15}{\log 7}$, and the boundary is $V_{0}$. By [11, Proposition 7.1], we have

$$
R_{n}\left(p_{1}, p_{2}\right) \asymp R_{n}\left(p_{2}, p_{3}\right) \asymp R_{n}\left(p_{3}, p_{1}\right) \asymp \lambda^{n},
$$

(equality does not hold) with some $\lambda>1\left(\lambda \approx 6.6448\right.$ numerically). Hence $K_{1}$ has property $(\mathrm{E})$, and it follows from the convergence theorem (Theorem 5.3) that there is a self-similar local regular Dirichlet form on $K$ with renormalizing factor $\lambda$. However, we do not know the uniqueness of such Dirichlet form on $K_{1}$. In fact, in [11, Proposition 7.1], we applied a "reverse recursive method" and the fixed point theorem to obtain the existence of a local regular self-similar Dirichlet form, with renormalization factor $\lambda^{-1}$ and has domain $\mathcal{F}=B_{2, \infty}^{\sigma^{*}}$. But we do not know if the two Dirichlet forms will be identical or equivalent.

There are two other variants $K_{2}$ and $K_{3}$ (Figures 2,3) that are similar, $K_{2}$ has 13 maps with contraction ratio $\rho=\frac{1}{6}, K_{3}$ has 10 maps with $\rho=\frac{1}{5}$ (see [11]).

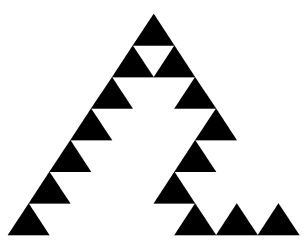

Figure 1. $K_{1}$.

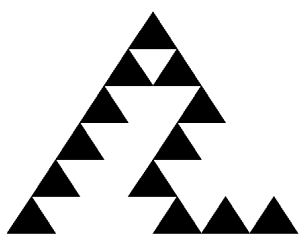

Figure 2. $K_{2}$.

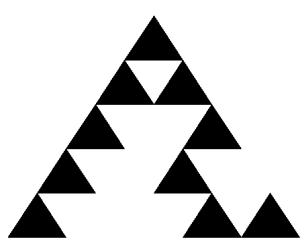

Figure $3 . K_{3}$.

Another interesting example studied in detail in [11, Section 6.2] is the selfsimilar set $K$ in Figure 4 that is generated by 17 similitudes with $\rho=\frac{1}{7}$. We call it the Sierpinski sickle. It also has boundary $V_{0}=\left\{p_{i}\right\}_{i=1}^{3}$. Unlike the previous cases, it was shown that

$$
R_{n}\left(p_{1}, p_{2}\right) \asymp R_{n}\left(p_{2}, p_{3}\right) \asymp\left(\frac{17}{2}\right)^{n}, \quad R_{n}\left(p_{3}, p_{1}\right) \asymp 7^{n} .
$$

This gives us two critical exponents; besides $\sigma^{*}$, there is another $\sigma^{\#}$ defined by

$$
\sigma^{\#}=\sup \left\{\sigma: B_{2, \infty}^{\sigma} \text { contains non-constant functions }\right\} \text {. }
$$

It was shown that $B_{2, \infty}^{\sigma^{*}}$ is dense in $\left(C(K),\|\cdot\|_{\infty}\right)$ in the uniform norm, and for $\sigma^{*}<\sigma \leq \sigma^{\#}, B_{2, \infty}^{\sigma}$ is dense in $L^{2}(K, \mu)$, but not dense in $\left(C(K),\|\cdot\|_{\infty}\right)$. Noting that at $\sigma^{\#}$, by examining the proof of Theorem 5.2 carefully, and take the $\Gamma$-limit of $\left(\sigma^{\#}-\sigma\right) \mathcal{E}_{2,2}^{\sigma}$ as $\sigma \uparrow \sigma^{\#}$, we can obtain a closed form truly in the wide sense which has domain $B_{2, \infty}^{\sigma^{\#}}$. While for the $\sigma^{*}$, clearly $B_{2, \infty}^{\sigma^{*}}$ does not admit a local regular Dirichlet form (otherwise, $\sigma^{*}=\sigma^{\#}$ ). We do not know the $\Gamma$-limit of the Dirichlet forms $\left(\sigma^{*}-\sigma\right) \mathcal{E}_{2,2}^{\sigma}$ at the critical exponent $\sigma^{*}$. On the other hand, by using the "reverse recursive technique" again, we can construct a regular self-similar Dirichlet form on $K$, but the domain is different from $B_{2, \infty}^{\sigma^{*}}$.

We remark that the self-similar Dirichlet form from the $\Gamma$-convergence is by taking convergent subsequence (Theorem 5.3), hence the uniqueness is not clear. Although the energy form is self-similar, it does not need to have the "harmonic structure", which is commonly used with the fixed point theorem to construct local regular 
Dirichlet forms (Kigami [16, Chapter 3]). In the first example, the Dirichlet form obtained from the reverse recursive construction has harmonic structure, and has the same domain as the one from the $\Gamma$-limit, but we still cannot conclude that they are equal. In general, the reverse recursive construction might not yield such Dirichlet form. Note also that there is diffusive approach to study the convergence ([22] and [17]). Over there, they assumed the existence of some two-sided heat kernel estimates of the diffusive processes first, then use them to construct approximating sequences. In our consideration, we do not put any assumption on the limiting Dirichlet form, which is a crucial point in our approach (see the remark at the end).
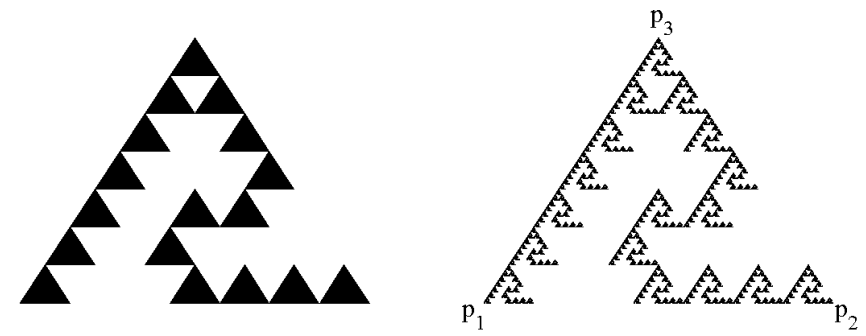

Figure 4. The Sierpinski sickle $K$.

For the sufficient condition for property (E) in Theorem 6.1, we basically rely on the resistance network technique on the p.c.f. sets. It is not clear what role the geometric properties of the p.c.f. sets play on the convergence at the critical exponents of the two classes of function spaces.

Q1. Find geometric condition of the p.c.f. sets (or more general $\alpha$-sets) for the $\Gamma$-convergence of $[\cdot]_{B_{2,2}^{\sigma}}^{2}$ to $[\cdot]_{B_{2, \infty}^{\sigma^{*}}}^{2}$.

For the energy form $(\mathcal{E}, \mathcal{F})$ satisfies the energy self-similar identity

$$
\mathcal{E}[u]=\sum_{i=1}^{N} \tau_{i}^{-1}\left[u \circ F_{i}\right], \quad u \in \mathcal{F},
$$

where the $\tau_{i}$ are not all equal, we need to consider a modified Besov space on the metric measure space $\left(K, d_{\mathbf{r}}, \nu\right)$ where $d_{\mathbf{r}}$ is the resistance metric on $K$, and $\nu$ is the self-similar measure $\left\{\tau_{i}^{s}\right\}_{i=1}^{N}$ with $\sum_{i=1}^{N} \tau_{i}^{s}=1[9,13,16]$. Also in [17], they actually considered the $\Gamma$-convergence of the Besov spaces on metric measure spaces equipped with doubling measures.

Q2. Can we extend the convergence theorem (Theorem 1.4) to these more general settings?

Our final remark is that the Dirichlet form $\mathcal{E}_{2,2}^{\sigma}$ is rather easy to obtain, but at the critical exponent for $\mathcal{E}_{2, \infty}^{\sigma^{*}}$, the situation is more difficult. In particular the Dirichlet form on $B_{2, \infty}^{\sigma^{*}}$ is not known to exist in general. It is seen that a further investigation of the convergence theorem (Theorem 5.3), and finding a more general condition than property (E) are important and interesting, and will lead to more knowledge on this open problem.

\section{Appendix}

A. Proof of Proposition 2.4. We will make use of a result [7]: if a selfsimilar measure $\mu$ on $K$ and is not an atom, then for any hyperplane $H$ in $\mathbf{R}^{d}$, either $\mu(H \cap K)=0$ or $H \supseteq K$. 
Proof of Proposition 2.4. Suppose the conclusion of the theorem does not hold, then there exists a ball $B(x, r) \subset \mathbf{R}^{d}$ such that $\delta:=\mu(\partial B(x, r))>0$. By the definition of self-similar measure, we have

$$
\mu(\partial B(x, r))=\sum_{|\omega|=n} p_{\omega} \cdot \mu\left(F_{\omega}^{-1}(\partial B(x, r))\right),
$$

where $p_{\omega}=p_{i_{1}} \cdots p_{i_{n}}$, It follows that for any $n>0$, there exists a word $\omega_{n}$ with $\left|\omega_{n}\right|=n$ such that

$$
\mu\left(F_{\omega_{n}}^{-1}(\partial B(x, r))\right) \geq \delta .
$$

Let $B(0, \eta)$ be a ball containing $K$, then $F_{\omega_{n}}^{-1}(\partial B(x, r)) \cap B(0, \eta) \neq \emptyset$. As $F_{\omega_{n}}^{-1}(\partial B(x$, $r))=\partial B\left(F_{\omega_{n}}^{-1}(x), \rho_{\omega_{n}}^{-1} r\right)$, the radii of the balls increases to $\infty$ as $n \rightarrow \infty$. By the smoothness of the sphere, there exists a hyperplane $H_{n}$ such that the Hausdorff distance of $F_{\omega_{n}}^{-1}(\partial B(x, r)) \cap B(0, \eta)$ and $H_{n} \cap B(0, \eta)$ is small. By the compactness of the family of compact sets in the Hausdorff metric, there exists a subsequence $\left\{n_{k}\right\}$ and a hyperplane $H$,

$$
F_{\omega_{n_{k}}}^{-1}(\partial B(x, r)) \cap B(0, \eta) \rightarrow H \cap B(0, \eta) .
$$

This implies

$$
\mu(H) \geq \liminf _{n \rightarrow \infty} \mu\left(F_{\omega_{n_{k}}}^{-1}(\partial B(x, r))\right) \geq \delta .
$$

By the result stated in the beginning, we conclude that $H \supset K$. This is a contradiction.

B. Proof of Proposition 2.5. We quote from [6, Proposition 2.4] that under the p.c.f. assumption, if $p \in K_{i} \cap K_{j}$, then $p$ has two representations $i \theta_{1} \dot{\xi}, j \theta_{2} \dot{\eta} \in \Sigma^{\infty}$, i.e.,

$$
\pi\left(i \theta_{1} \dot{\xi}\right)=\pi\left(j \theta_{2} \dot{\eta}\right)=p
$$

where $\dot{\xi}, \dot{\eta}$ are recurrent words; we can also choose $\theta_{1}$ and $\theta_{2}$ to be the shortest words respectively so that the above holds.

Lemma 8.1. Suppose the IFS $\left\{F_{i}\right\}_{i=1}^{N}$ has the p.c.f. property, and $p \in K_{i} \cap$ $K_{j}, i \neq j$. Then

(i) there exists $k_{0}$ (independent of $i, j$ ) such that for $|\omega|=\left|\omega^{\prime}\right|>k_{0}, K_{i \omega} \cap K_{j \omega^{\prime}}$ contains at most one point.

(ii) suppose $\omega, \omega^{\prime}$ are such that $K_{\omega} \cap K_{\omega^{\prime}}=\emptyset$, and

$$
\omega=i \theta_{1} \underbrace{\xi \cdots \xi}_{n_{2} k} \xi^{\prime}:=i \theta_{1} \xi^{n_{2} k} \xi^{\prime}, \quad \omega^{\prime}=j \theta_{2} \underbrace{\eta \cdots \eta}_{n_{1} k} \eta^{\prime}:=j \theta_{2} \eta^{n_{1} k} \eta^{\prime}
$$

where $i \theta_{1} \dot{\xi}, j \theta_{2} \dot{\eta}$ are as in (8.1) with $|\xi|=n_{1},|\eta|=n_{2}$, and $\left|\xi^{\prime}\right|,\left|\eta^{\prime}\right| \leq n_{1} n_{2}$. Then

$$
\operatorname{dist}\left(K_{\omega}, K_{\omega^{\prime}}\right)=\rho^{n_{1} n_{2} k} \operatorname{dist}\left(K_{i \theta_{1} \xi^{\prime}}, K_{j \theta_{2} \eta^{\prime}}\right) .
$$

Proof. The p.c.f. assumption implies that the intersection $K_{i} \cap K_{j}$ is a finite set for any $i \neq j$. Let $k_{i, j}$ be sufficiently large such that for any $\omega, \omega^{\prime} \in \Sigma^{*}$ with $|\omega|,\left|\omega^{\prime}\right| \geq k_{i, j}, K_{i \omega} \cap K_{j \omega^{\prime}}$ contains at most one point. Since there are only finitely many such pair $i, j$, we take $k_{0}=\max _{i, j}\left\{k_{i, j}\right\}$ and it will satisfy the assertion in (i).

To prove (ii), we let $\bar{p}_{\xi}=\lim _{n \rightarrow \infty} K_{\xi^{n}}$. Then $\bar{p}_{\xi}$ is a fixed point of $F_{\xi}$, and by observing that $|\xi|=n_{1}$, we have for any $n \geq 1, F_{\xi^{n}}(x)=\rho^{n_{1} n}\left(x-\bar{p}_{\xi}\right)+\bar{p}_{\xi}$. Also let $\bar{p}_{i \theta_{1}}$ be the fixed point of $F_{i \theta_{1}}$, then by (8.1), we have

$$
p=\rho^{\left|\theta_{1}\right|+1}\left(\bar{p}_{\xi}-\bar{p}_{i \theta_{1}}\right)+\bar{p}_{i \theta_{1}} .
$$


It follows that

$$
\begin{aligned}
K_{i \theta_{1} \xi^{n^{2} k} \xi^{\prime}}-p & =\rho^{\left|\theta_{1}\right|+1}\left(K_{\xi^{n_{2} k} \xi^{\prime}}-\bar{p}_{i \theta_{1}}\right)+\bar{p}_{i \theta_{1}}-p \\
& =\rho^{\left|\theta_{1}\right|+1}\left(K_{\xi^{n_{2} k} \xi^{\prime}}-\bar{p}_{\xi}\right) \\
& =\rho^{\left|\theta_{1}\right|+1+n_{1} n_{2} k}\left(K_{\xi^{\prime}}-\bar{p}_{\xi}\right) \\
& =\rho^{n_{1} n_{2} k}\left(K_{i \theta_{1} \xi^{\prime}}-p\right) .
\end{aligned}
$$

Similarly, we have $K_{j \theta_{2} \eta^{n_{1} k} \eta^{\prime}}-p=\rho^{n_{1} n_{2} k}\left(K_{j \theta_{2} \eta^{\prime}}-p\right)$. This implies (8.2).

Proof of Proposition 2.5. Note that the number of triples $(i, j, p)$ in Lemma 8.1 is finite, and for each $(i, j, p)$, the total number of words $i \theta_{1} \xi^{\prime}, j \theta_{2} \eta^{\prime}$ are finite. By (8.2), there exists $c_{1}>0$, such that for $|\omega|=\left|\omega^{\prime}\right|=m$ with the form as in Lemma 8.1(ii) and $K_{\omega} \cap K_{\omega^{\prime}}=\emptyset$, then

$$
\operatorname{dist}\left(K_{\omega}, K_{\omega^{\prime}}\right) \geq c_{1} \rho^{m}
$$

Next, let $k_{0}$ be as in Lemma 8.1(i). Consider any two finite words $\omega$ and $\omega^{\prime}$ of length $m \geq 2 k_{0}$, and $K_{\omega} \cap K_{\omega^{\prime}}=\emptyset$, we let $\left.\omega\right|_{n}$ and $\left.\omega^{\prime}\right|_{n}$ denote the initial segments of $\omega$ and $\omega^{\prime}$ of length $n$. We observe the following two cases:

(i) If $K_{\left.\omega\right|_{k_{0}}} \cap K_{\left.\omega^{\prime}\right|_{k_{0}}}=\emptyset$, then we simply have

$$
\operatorname{dist}\left(K_{\omega}, K_{\omega^{\prime}}\right) \geq \operatorname{dist}\left(K_{\left.\omega\right|_{k_{0}}}, K_{\omega^{\prime} \mid k_{0}}\right) \geq c \geq c_{2} \rho^{m}
$$

where $c>0$ is the minimum of the distances of disjoint cells with word length $\leq k_{0}$.

(ii) Otherwise, let $n_{0}$ be the smallest $k_{0}<n \leq m$ such that

$$
K_{\left.\omega\right|_{n_{0}}} \cap K_{\left.\omega^{\prime}\right|_{n_{0}}}=\emptyset .
$$

As $k_{0} \leq n<n_{0}$, by Lemma 8.1(i), we see that $K_{\left.\omega\right|_{n}} \cap K_{\left.\omega^{\prime}\right|_{n}}$ has exactly one intersection point, then $K_{\left.\omega\right|_{n_{0}}}$ and $K_{\left.\omega^{\prime}\right|_{n_{0}}}$ has the form as in Lemma 8.1(ii). By (8.3), we have

$$
\operatorname{dist}\left(K_{\omega}, K_{\omega^{\prime}}\right) \geq \operatorname{dist}\left(K_{\left.\omega\right|_{n_{0}}}, K_{\left.\omega^{\prime}\right|_{n_{0}}}\right) \geq c_{1} \rho^{n_{0}} \geq c_{1} \rho^{m} .
$$

Let $c_{0}=\min \left\{c_{1}, c_{2}\right\}$, then condition $(\mathrm{H})$ follows.

Acknowledgement. The authors are indebted to the referees for many useful comments and bringing their attention to some relevant references, which help improving the presentation of the paper. They also would like to thank Professor D.-J. Feng for providing the proof of Proposition 2.4, and Professor J. Hu and Drs. S.-L. Kong, M. Yang for many valuable discussions.

\section{References}

[1] Barlow, M., and R. BASs: The construction of Brownian motion on the Sierpiński carpet. Ann. Inst. H. Poincaré Probab. Stat. 25, 1989, 225-257.

[2] Barlow, M., and R. BAss: Transition densities for Brownian motion on the Sierpiński carpet. - Probab. Theory Related Fields 91, 1992, 307-330.

[3] Bodin, M.: Discrete characterisations of Lipschitz spaces on fractals. - Math. Nachr. 282, 2009, 26-43.

[4] Bourgain, J., H. Brezis, and P. Mironescu: Another look at Sobolev spaces. - Optimal Control and Partial Differential Equation, IOS, Amsterdam, 2001, 439-455.

[5] Dal Maso, G.: An introduction to $\Gamma$-convergence. - Progr. Nolinear Differential Equations Appl. 8, Birkhäusser, 1993.

[6] Deng, Q.-R., and K.-S. LAU: Open set condition and post-critically finite self-similar sets. Nonlinearity 21, 2008, 1227-1232. 
[7] Elekes, M., T. Keleti, and A. Máthé: Self-similar and self-affine sets: measure of the intersection of two copies. - Ergodic Theory Dynam. Systems 30, 2010, 399-440.

[8] Feng, D.-J.: Private communication.

[9] Grigor'yan, A., J. Hu, and K.-S. LAU. Heat kernels on metric-measure spaces and an application to semilinear elliptic equations. - Trans. Amer. Math. Soc. 355, 2003, 2065-2095.

[10] Grigor'Yan, A., and M. YAng: Local and non-local Dirichlet forms on Sierpinski carpet. Trans. Amer. Math. Soc. (to appear).

[11] Gu, Q., and K.-S. LAU: Dirichlet forms and critical exponents on fractals. - Trans. Amer. Math. Soc. (to appear).

[12] Gu, Q., K.-S. LAU, and J. XIAO: Resistance estimates and Dirichlet forms on some classes of non-p.c.f. fractals. - In preparation.

[13] Hu, J., and X. S. WANG: Domains of Dirichlet forms and effective resistance estimates on p.c.f. fractals. - Studia Math. 177, 2006, 153-172.

[14] Jonsson, A.: Brownian motion on fractals and function spaces. - Math. Zeit. 222, 1996, 495-504.

[15] Jonsson, A., and H. Wallin: Function spaces on subsets of $R^{n}$. - Math. Reports 2, Acad. Publ., Harwood, 1984.

[16] Kigami, J.: Analysis on fractals. - Cambridge Univ. Press, 2001.

[17] Kumagai, T., and K.-T. Sturm: Construction of diffusion processes on fractals, $d$-sets, and general metric measure spaces. - J. Math. Kyoto Univ. 45, 2005, 307-327.

[18] Kusuoka, S., and X. Y. Zhou: Dirichlet forms on fractals: Poincaré constant and resistance. - Probab. Theory Related Fields 93, 1992, 169-196.

[19] LAU, K.-S., and X.-Y. WANG: Self-similar sets as hyperbolic boundaries. - Indiana U. Math. J. 58, 2009, 1777-1795.

[20] Mosco, U.: Composite media and asymptotic Dirichlet forms. - J. Funct. Anal. 123, 1994, 368-421.

[21] Moser, J.: On Harnack's theorem for elliptic differential equations. - Comm. Pure Appl. Math. 14, 1961, 577-591.

[22] Pietruska-PaŁuba, K.: Limiting behvior of Dirichlet forms for stable processes on metric spaces. - Bull. Pol. Acad. Sci. Math. 56, 2008, 257-266.

[23] Pietruska-PaŁuba, K., and A. Stós: Poincaré inequality and Hajłasz-Sobolev spaces on nested fractals. - Studia Math. 218, 2013, 1-26.

[24] SAвот, C.: Existence and uniqueness of diffusions on finitely ramified self-similar fractals. Ann. Sci. École Norm. Sup. (4) 30, 1997, 605-673.

[25] Strichartz, R.: Function spaces on fractals. - J. Funct. Anal. 198, 2003, 43-83.

[26] Strichartz, R.: Differential equations on fractals: a tutorial. - Princeton Univ. Press, 2006.

[27] Tetenov, A., K. Kamalutdinov, and D. Vaulin: Self-similar Jordan arcs which do not satisfy OSC. - arXiv:1512.00290.

[28] Triebel, H.: Fractals and spectra, related to Fourier analysis and functional analysis. - Mono. Math. 91, Birkhäuser, 1997.

[29] YAng, M.: The local regular Dirichlet form on the Sierpinski gasket via the average method. - Preprint. 